\title{
Sodium Tanshinone IIA Sulfonate Promotes Spinal Cord Injury Repair by Inhibiting BSCB Disruption In Vitro and In Vivo
}

\section{Dan Luo}

The Second Affiliated Hospital of Shandong First Medical University

\section{Xing Li}

Research Laboratory of Spine Degenerative Disease, The Second Affiliated Hospital of Guangzhou University of Chinese Medicine, Guangzhou 510120, China;2. Laboratory of Osteology and Traumatology of Traditional Chinese Medicine, Lingnan Medical Research

\section{Jiheng Zhan}

Research Laboratory of Spine Degenerative Disease, The Second Affiliated Hospital of Guangzhou University of Chinese Medicine, Guangzhou 510120, China;2. Laboratory of Osteology and Traumatology of Traditional Chinese Medicine, Lingnan Medical Research

\section{Yonghui Hou}

Research Laboratory of Spine Degenerative Disease, The Second Affiliated Hospital of Guangzhou University of Chinese Medicine, Guangzhou 510120, China;2. Laboratory of Osteology and Traumatology of Traditional Chinese Medicine, Lingnan Medical Research

Jiyao Luan

Research Laboratory of Spine Degenerative Disease, The Second Affiliated Hospital of Guangzhou University of Chinese Medicine, Guangzhou 510120, China;2. Laboratory of Osteology and Traumatology of Traditional Chinese Medicine, Lingnan Medical Research

\section{Dingkun Lin ( $\nabla$ lindingkuntcm@126.com )}

Research Laboratory of Spine Degenerative Disease, The Second Affiliated Hospital of Guangzhou University of Chinese Medicine, Guangzhou 510120, China;2. Laboratory of Osteology and Traumatology of Traditional Chinese Medicine, Lingnan Medical Research

\section{Research}

Keywords: Sodium tanshinone IIA Sulfonate, Blood Spinal Cord Barrier, Spinal Cord Injury

Posted Date: December 2nd, 2020

DOl: https://doi.org/10.21203/rs.3.rs-117487/v1 
License: (c) (i) This work is licensed under a Creative Commons Attribution 4.0 International License. Read Full License 


\section{Abstract}

Background:Spinal cord injury (SCl) leads to microvascular damage and the destruction of blood spinal cord barrier (BSCB), which progresses to secondary injuries like apoptosis and necrosis of neurons and glia, culminating in permanent neurological deficits. BSCB restoration is the primary goal of SCI therapy, although very few drugs can repair the damaged barrier structure and permeability. Sodium tanshinone IIA sulfonate (STS) is commonly used to treat cardiovascular disease. We found that STS restored BSCB integrity and promoted microvessel recovery 7 days after $\mathrm{SCl}$ in a mouse model. However, the therapeutic effects of STS on damaged BSCB in the early stage of SCl remained uncertain.

Methods: we exposed spinal cord microvascular endothelial cells (SCMECs) to $\mathrm{H} 2 \mathrm{O} 2$ and treated them with different doses of STS. The mice received intraperitoneal injection of STS after SCl in vivo model. Spinal cord tissue was taken 1 and 3d post-SCI. HE, Nissl staining, BSCB permeability, and the expression levels of tight junction (TJ) and adherens junction (AJ), MMP2, MMP9, NeuN, and C-caspase-3 were analyzed.

Results: In addition to protecting the cells from $\mathrm{H}_{2} \mathrm{O}_{2}$-induced apoptosis, STS also reduced cellular permeability. In the in vivo model of SCI as well, STS reduced BSCB permeability, relieved tissue edema and hemorrhage, suppressed MMPs activation and prevented TJ and AJ the loss of proteins.

Conclusions:Our findings indicate that STS treatment promotes SCl recovery, and should be investigated further as a drug candidate against traumatic SCl.

\section{Background}

Spinal cord injury (SCl) can cause permanent functional defects in the limbs through primary and secondary injuries[1,2]. Primary injury is the result of the initial trauma that leads to structural disturbance, whereas secondary injuries consist of inflammation, apoptosis, axonotmesis and formation of glial scars, which eventually culminate to progressive secondary hemorrhage in the central nervous system (CNS) $[3,4]$. The blood spinal cord barrier (BSCB) has a similar function to that of the blood-brain barrier (BBB) $[5,6]$. It comprises of specialized endothelial cells that are attached via tight junctionassociated (TJ) and adherens junction-associated (AJ) protein complexes, and enclosed by the end feet of astrocytes[7]. The BSCB blocks the entry of circulating toxins and pathogens into the CNS[8], and SClinduced damage to the BSCB allows influx of endogenous inflammatory factors and pathogens into the spinal cord tissues, which further aggravates SCI $[9,10]$. Therefore, BSCB integrity is crucial for motor function recovery after $\mathrm{SCl}$.

The spinal cord microvascular endothelial cells (SCMECs) are essential for maintaining the structural integrity and normal physiological function of the BSCB[11-13]. The impermeability of the BSCB depends on the TJ and AJ proteins that are expressed by the SCMECs, and form a barrier which keeps solutes and cells from entering into the inner environment[14]. The activation of matrix metalloproteases (MMPs), especially MMP-9 and MMP-2, is the key pathological basis of BSCB disruption[15-17]. MMP9 
causes proteolytic degradation of the BBB, and blocking MMP9 activity can attenuate vascular permeability[18].In fact, restoring BSCB integrity is the primary therapeutic approach for SCl.

Tanshinone IIA is a major bioactive component of Salvia miltiorrhiza, which is used in several traditional Chinese medicine formulations[19]. However, the poor water solubility and low bioavailability of Tanshinone IIA limits its clinical applications[20, 21]. Sodium tanshinone IIA sulfonate (STS) has greater water solubility and can effectively promote blood circulation, remove blood stasis, and activate channels and collateral[22, 23]. STS is widely used for treating coronary artery disease, angina pectoris and myocardial infarction[24]. Studies show that STS can not only exert neuroprotective effects but also repair the BBB in stroke patients[25-27]. Our preliminary results indicated that STS restored BSCB function and rescued microvessels 7 days after SCI[28]. However, the therapeutic effects of STS in the early stages post-SCl remain to be elucidated.

To this end, we analyzed the effects of STS on $\mathrm{H}_{2} \mathrm{O}_{2}$-treated SCMECs, and found that it restored BSCB integrity, prevented loss of TJ and AJ proteins, inhibited MMPs activation, and exerted neuroprotective effects in the early stage of $\mathrm{SCl}$.

\section{Materials \& Methods}

\subsection{Reagents}

Endothelial growth medium 2 was purchased from LONZA, (California, USA). DMEM, fetal bovine serum (FBS) and trypsin were purchased from Gibco (New York, USA). Cell counting kit-8 (CCK-8) and BCA assay kit were bought from Beyotime Biotechnology (Shanghai, China). Evans Blue and fluorescein isothiocyanate (FITC)-dextran were purchased from Aladdin (Shanghai, China), and STS (purity $>99 \%$ using HPLC) from Chengdu Herbpurify Co. Ltd. (Chengdu, China).

\subsection{SCMECs culture and viability test}

SCMECs were maintained in EGM-2 supplemented with 5\% FBS and antibiotics (1\% penicillin and 1\% streptomycin) under $5 \% \mathrm{CO}_{2}$ at $37^{\circ} \mathrm{C}$. The medium was replaced every 2 days. The cells were cultured in the presence of 25-400 $\mu \mathrm{M} \mathrm{H}_{2} \mathrm{O}_{2}$ for $12 \mathrm{~h}$, and $10 \mu \mathrm{l} \mathrm{CCK}-8$ reagent was added to each well. After incubating for $2 \mathrm{~h}$, the absorbance at $450 \mathrm{~nm}$ was detected using a microplate reader (Bio Red, USA) to determine the proportion of viable cells. The cells were exposed to the suitable $\mathrm{H}_{2} \mathrm{O}_{2}$ concentration, followed by $0,5,10$ and $20 \mu \mathrm{M}$ STS. Cell viability was evaluated by the CCK-8 assay as described. All experiments were performed in triplicate.

\subsection{Cell permeability measurement}

The STS-treated cells were seeded into Transwell chambers containing $0.4 \mu \mathrm{m}$ pore size membrane in a 24 -well plate at the density of $2 \times 10^{4}$ cells/200 $\mu \mathrm{l} /$ well. The lower chambers were filled with $500 \mu \mathrm{l}$ medium, and the cells were cultured for $24 \mathrm{~h}$. Once the cells adhered, the medium was replaced with 
sugar-free medium and the cells were incubated for $12 \mathrm{~h}$ with $200 \mu \mathrm{M} \mathrm{H}_{2} \mathrm{O}_{2}$ in the dark. To each well, $4 \mu \mathrm{l}$ FITC-dextran $(50 \mu \mathrm{g} / \mu \mathrm{l})$ was added and the cells were incubated for $4 \mathrm{~h}$. Finally, $200 \mu \mathrm{l}$ medium was aspirated from each lower chamber, and fluorescence intensity (excitation wavelength $485 \mathrm{~nm}$, emission wavelength $520 \mathrm{~nm}$ ) was measured using a microplate reader.

\subsection{Establishment of SCI model}

Ten weeks old male C57BL/ 6 mice were provided by Guangdong Medical Laboratory Animal Center (Foshan, China, Certificate No.44005800011215). After anesthetizing with 1\% (w/v) pentobarbital sodium (40 mg/kg), the mice were subjected to laminectomy at the T9-T10 level to expose the spinal cord without damaging the dura. A contusion was made using a pneumatic impact device as described by Allen et al. For the sham operated controls, T10 laminectomy was performed without impact injury. All animals were treated with antibiotics to prevent local and urinary infection. Bladder massage was performed to facilitate defecation post-surgery if needed. The STS-treated group received intraperitoneal injection of $20 \mathrm{mg} / \mathrm{kg}$ STS $1 \mathrm{~h}$ after SCl and once daily thereafter, whereas the untreated model and sham-operated groups were injected with the same volume of $0.9 \%$ sodium chloride.

\subsection{Evans blue assay}

The permeability of the BSCB was assessed by the Evans blue dye extravasation method[29]. Briefly, the mice were injected intravenously with $150 \mu \mathrm{l}$ of $2 \%$ Evans blue dye in saline on days 1 and 3 post-SCl. The mice were anesthetized $3 \mathrm{~h}$ later and euthanized by intra-cardiac perfusion with PBS and $4 \%$ paraformaldehyde (PFA). The spinal cords were dissected, and cut into $30 \mu \mathrm{m}$ cross sectional slices using an Ultra-Thin Semiautomatic Microtome (Leica RM2255, Germany). The fluorescence intensity was observed under a microscope.

\subsection{Water content of spinal cord}

Spinal cords were extracted as described from five random mice per group on days 1 and 3 post-SCl. One centimeter-long pieces were cut from the injured site and weighed. The tissues were then dried in an oven at $60^{\circ} \mathrm{C}$ for $48 \mathrm{~h}$, and the dehydrated specimens were weighed. The water content of the spinal cord was calculated as (wet weight - dry weight)/wet weight.

\subsection{Tissue preparation}

Mice were anesthetized and perfused by cardiac puncture with $0.1 \mathrm{M}$ PBS and 4\% PFA on days 1 and 3 post-SCI. The spinal cords were removed, and some segments were embedded in OTC and cut into 10$\mu \mathrm{m}$-thick frozen sections, and others were fixed with $10 \%$ formaldehyde, embedded with paraffin and cut into 5 - $\mu$ m-thick sections. For molecular assays, the mice ( $n=5 /$ group per time point) were perfused with $0.1 \mathrm{M}$ PBS and $10 \mathrm{~mm}$ spinal cord segments including the lesion site were isolated and frozen at $-80^{\circ} \mathrm{C}$.

\subsection{Western blotting}

The protein content of cell lysates and tissue homogenates were measured using the BCA assay kit. Equivalent amounts of protein per sample were separated in 10\% SDS-PA gels, and transferred to PVDF 
membranes (Millipore). After blocking with $5 \%$ skim milk in $0.5 \%$ Tris-buffered saline Tween (TBST) for $1 \mathrm{~h}$ at room temperature, the membranes were incubated overnight with rabbit anti- $\beta$-catenin (1:1000; Abcam), rabbit anti-occludin (1:1000; Abcam), rabbit anti-Bcl2 (1:1000; Abcam), rabbit anti-Bax (1:1000; Cell Signaling Technology), mouse anti-c-caspase-3 (1:1000; Cell Signaling Technology), rabbit anti-MMP2 (1:1000; Abcam), rabbit anti-MMP-9 (1:1000; Abcam) and rabbit anti-GAPDH (1:1000; Cell Signaling Technology) primary antibodies at $4{ }^{\circ} \mathrm{C}$. The bands were then probed with horseradish peroxidase (HRP)conjugated rabbit/mouse secondary antibodies (1:1000; Abcam). The experiment was repeated thrice.

\subsection{Histopathology and immunofluorescence}

Paraffin-embedded sections were stained with hematoxylin and eosin (HE) and Nissl's dye according to standard protocols. Tissue cryosections and cells grown on glass coverslips were washed thrice with PBS, fixed with $4 \%$ PFA, permeabilized with $0.3 \%$ Triton X-100 and blocked with $10 \%$ goat serum. After incubating overnight with primary antibodies against occluding (1:200, Abcam), NeuN (1:200, Abcam) and C-caspase $3(1: 200, A b c a m)$ at $4{ }^{\circ} \mathrm{C}$, the samples were washed thrice with PBS, and probed with AlexaFluor 488 donkey anti-rabbit/mouse secondary antibodies (1:200) for $1 \mathrm{~h}$ at room temperature. The tissue sections or cells were rinsed again with PBS, counterstained with DAPI for 5 min, rinsed, and sealed with a coverslip. The slides were observed under a fluorescence microscope.

\subsection{Statistical analysis}

SPSS 24.0 software was used for statistical analysis. The data were expressed as mean \pm standard deviation (SD), and compared using unpaired Student's T-Test. P-values $<0.05$ were considered statistically significant.

\section{Results}

\subsection{STS protects SCMECs from $\mathrm{H}_{2} \mathrm{O}_{2}$-induced damage and restores barrier function}

The chemical structure of STS is shown in Fig. 1A. The SCMECs were treated with varying concentrations of $\mathrm{H}_{2} \mathrm{O}_{2}$, and $200 \mu \mathrm{M}$ was selected for subsequent experiments. As shown in Fig. 1B, STS increased the viability of the $\mathrm{H}_{2} \mathrm{O}_{2}$-treated cells in a dose-dependent manner. Furthermore, STS treatment also decreased the permeability of the SCMECs following $\mathrm{H}_{2} \mathrm{O}_{2}$ exposure, as indicated by reduced uptake of FITC-dextran (Fig. 1C). The TJ and AJ proteins between SCMECs maintain the barrier function of BSCB, and their loss is directly related to increased permeability[30]. As shown in Fig. $1 \mathrm{E}-\mathrm{G}, \mathrm{H}_{2} \mathrm{O}_{2}$ treatment significantly decreased the levels of occludin and $\beta$-catenin in SCMECs, and both were restored by STS in a dose-dependent manner. Overactivation of the MMPs in the extracellular matrix of injured spinal cord tissues degrades the BSCB and leads to a massive influx of white blood cells, which further aggravates the secondary injury[31]. Consistent with this, both MMP2 and MMP9 levels increased significantly after $\mathrm{H}_{2} \mathrm{O}_{2}$ treatment, and were reduced to near baseline levels by STS (Fig. 1H-K). Taken together, STS can 
reverse the $\mathrm{H}_{2} \mathrm{O}_{2}$-induced hyperpermeability of SCMECs by blocking MMP activation and maintaining the $\mathrm{TJ}$ and AJ proteins. Furthermore, $200 \mu \mathrm{M} \mathrm{H}_{2} \mathrm{O}_{2}$ markedly increased the expression of pro-apoptotic proteins such as Bax and cleaved Caspase-3 but reduced that of Bcl-2. In contrast, STS reversed the apoptotic program in the $\mathrm{H}_{2} \mathrm{O}_{2}$-treated cells in a dose-dependent manner (Fig. 2A-C). Consistent with this, TUNEL staining confirmed that STS reduced the percentage of apoptotic SCMECs following $\mathrm{H}_{2} \mathrm{O}_{2}$ exposure (Fig. 2D).

\subsection{STS maintains BSCB integrity after SCI by preventing MMP activation and loss of junction proteins}

BSCB disruption following SCI was evaluated by Evans blue extravasation and intraspinal hemorrhage assay. As shown in Fig. 3A-B, the injured spinal cords showed increased Evans blue dye extravasation on days 1 and 3 post-surgery compared to the sham-operated controls, indicating that $\mathrm{SCl}$ triggered BSCB disruption. However, STS treatment sharply reduced the extent of Evans blue dye extravasation on both time points, which suggested a protective effect of STS on the BSCB. The total amount of Evans blue in the spinal cord tissues and its fluorescence intensity in the sham-operated, vehicle-treated and STStreated groups were also quantified and were consistent with the images (Fig. 3C-D). MMP-2 and MMP-9 play a key role in BSCB disruption post SCl by degrading the supporting ECM. As shown in Fig. 3E-G, MMP-2 and MMP-9 protein levels markedly increased on days 1 and 3 after injury compared to the shamoperated control, and were downregulated by STS. In addition, the in-situ expression of occludin and $\beta$ catenin were also low in the injured spinal cord, and restored by STS treatment (Fig. 5A-D). Thus, STS protects the BSCB after SCl by preventing loss of junction proteins and inhibiting MMP activity.

\subsection{STS reduced secondary hemorrhage and edema after $\mathrm{SCl}$}

Surface bleeding was observed in the injured spinal cords after 1 and 3 days of contusion, and was markedly alleviated in the STS-treated group versus the vehicle-treated control (Fig. 4A-B). HE staining of the spinal cord lesions indicated few inflammatory cells in the sham-operated group, along with normal tissue structure, proper cellular arrangement in each layer, and absence of any swelling or bleeding. In contrast, the injured spinal cords had extensive hemorrhagic areas, swollen and disrupted nerve cells, massive infiltration of inflammatory cells, widened interstitial spaces and severe edema. STS treatment not only reduced the hemorrhagic areas but also alleviated the other pathological changes (Fig. 4C). Furthermore, the water content in the spinal cord tissues also increased significantly after injury compared to the sham-operated controls, and was decreased by STS (Fig. 4D).

\subsection{STS reduced neuronal apoptosis in the spinal cord lesions}

Any injury to the spinal cord triggers apoptosis around the trauma epicenter[32-34]. Consistent with this, induction of $\mathrm{SCl}$ in our model significantly increased the levels of Bax protein and downregulated that of Bcl-2 on days 1 and 3 d post-injury. In contrast, STS treatment enhanced Bcl-2 expression and inhibited 
Bax (Fig. 6A-B). TUNEL staining also showed a marked increase in the number of apoptotic cells after injury, which was alleviated by STS treatment (Fig. 6C-D). Furthermore, co-staining for NeuN (green) and cleaved caspase-3 (red) indicated that the number of apoptotic neurons in particular increased after $\mathrm{SCl}$, whereas STS intervention led to a decline in neuronal apoptosis (Fig. 7A). The total cleaved caspase-3 protein level also decreased significantly in the injured spinal cords from the STS-treated animals (Fig. 7B-C). Consistent with the above, SCl led to a significant loss of motor neurons as revealed by Nissl staining, which was recovered by STS (Fig. 7D). Taken together, STS can alleviate the pathological effects of SCl by reversing neuronal loss.

\section{Discussion}

Spinal cord injury (SCl) often leads to permanent loss of limb function defect due to disruption of the BSCB and the ensuing secondary injuries[35, 36]. The BSCB is a semi-permeable interface of blood vessels that surrounds the spinal cord, and consists of the basement membrane, endothelial cells, pericytes, and astrocytes endplate foot processes[37]. The BSCB protects the spinal cord microenvironment from both endogenous and exogenous factors[38, 39]. Functional impairment of the $\mathrm{BSCB}$ is a key pathological event in $\mathrm{SCl}$, and leads to edema and secondary nerve injury. The disrupted BSCB allows blood flow into the spinal cord, which increases the risk of infection and inflammation. In addition, the infiltrating blood cells may produce neurotoxic substances that damage the neurons and synapses, eventually leading to glial cell and neuronal apoptosis. BSCB dysfunction caused by endovascular treatment increase patient mortality, while its functional recovery can significantly reduce secondary nerve injury after SCl. Sarah et al. showed that the BSCB is damaged within an hour of SCl, and remains unrepaired for 5 days. Peak BSCB permeability is usually observed 24 hours after $\mathrm{SCl}$, and early functional recovery of BSCB can significantly alleviate secondary injury[40].

STS is a water-soluble derivative of Tanshinone IIA, one of the most pharmacologically active monomers extracted from Salvia miltiorrhiza. Both STS and Tanshinone IIA can induce vasodilation, inhibit inflammatory response and prevent atherosclerosis and cardiac injury[41-43]. Jun Cheng et al.[44] found that STS prevented LPS-induced proinflammatory cytokine expression and secretion by inhibiting the NFKB signaling pathway in HUVECs. However, the potential therapeutic effect of STS on BSCB disruption in the early stages of $\mathrm{SCl}$ have not been demonstrated so far. We established an in vitro model of SClrelated BSCB disruption by exposing the SCMECs to $\mathrm{H}_{2} \mathrm{O}_{2}$, and evaluated the effects of STS on the viability and permeability of SCMECs. STS not only decreased the uptake of FITC-dextran but also protected the SCMECs from $\mathrm{H}_{2} \mathrm{O}_{2}$-induced toxicity.

MMP-9 and MMP-2 mediate BSCB breakdown during SCI by degrading the AJ and TJ proteins, and suppressing MMP-9 activity inhibits vascular permeability[45]. MMP-9 activity increases rapidly within the first $24 \mathrm{~h}$ post-injury and its peak levels correlate with maximum BSCB disruption[46]. In addition, increased level of MMP-2 is also related to the degree of SCI[47]. STS treatment reversed the $\mathrm{H}_{2} \mathrm{O}_{2}$ induced increase in MMPs and reduction in occludin and $\beta$-catenin synthesis in the SCMECs, and also inhibited the apoptosis program. Taken together, STS can maintain the functional integrity of SCMECs 
under pathological stimuli. In the in vivo model of SCl as well, $20 \mathrm{mg} / \mathrm{kg}$ STS reduced the synthesis of both MMPs and increased the levels of junction proteins. The permeability of the BSCB was assessed using Evans blue, which has a high affinity for serum albumin and therefore cannot pass through the BSCB under normal conditions. However, a damaged barrier allows the tracer dye to stain the spinal cord tissues. We found that SCI induction significantly increased Evan blue extravasation, which was reduced by STS treatment. Thus, STS can preserve the structural integrity of the BSCB after SCI by inhibiting the proteolytic action of MMPs and promoting synthesis of TJ and AJ proteins.

$\mathrm{SCl}$ triggers extensive neuronal apoptosis and vascular damage, which leads to the loss of motor neurons and a reduction in the number of blood vessels[48-50]. In addition, the progressive failure of capillary function leads to hemorrhaging[51]. The number of apoptotic neurons in the injured spinal cord declined sharply after STS administration, which also reduced capillary fragmentation and internal bleeding compared to the vehicle-treated control. To summarize, STS reduces the permeability of damaged BSCB in the early stage of $\mathrm{SCl}$, alleviates secondary injuries and hemorrhage, and accelerates functional recovery.

\section{Conclusion}

STS prevents BSCB disruption and hemorrhage in the early stages of $\mathrm{SCl}$ by inhibiting MMPs activation and promoting synthesis of TJ and AJ proteins. Furthermore, STS treatment protected the motor neurons from apoptosis and accelerated functional recovery. This is the first report to demonstrate the therapeutic efficacy of STS in the early stages of SCl, and should be investigated further as a candidate drug against traumatic SCl.

\section{Abbreviations}

SCl: Spinal cord injury

BSCB : Blood spinal cord barrier

STS: Sodium tanshinone IIA sulfonate

SCMECs : Spinal cord microvascular endothelial cells

TJ : Tight junction

AJ : Adherens junction

BBB : Blood-brain barrier

MMPs : Matrix metalloproteases

DMEM $\triangle$ Dulbecco's modified eagle medium 


\section{Declarations}

\section{Ethics approval and consent to participate}

All animal experiments were approved by the Institutional Animal Care and Use Committee of Guangzhou University of Chinese Medicine and performed according to the "NIH Guide for the Care and Use of Laboratory Animals". No work on humans was included in this manuscript.

\section{Availability of data and materials}

The primary data for this study is available from the authors on direct request.

\section{Consent for publication}

All authors have agreed to publish this article.

\section{Competing interests}

The authors declare that they have no competing interests.

\section{Authors' contributions}

DL\XL and DK contributed to conception and design. DL and XL analyzed the data. DL drafted the manuscript. $\mathrm{XL}, \mathrm{JZ}, \mathrm{YH}$, and $\mathrm{JL}$ contributed critical revision of the manuscript. All authors read and approved the final manuscript.

\section{Funding}

This work was supported by the National Natural Science Foundation of China (No. 81673992)

\section{Acknowledgements}

There are no acknowledgements except for funding sources already mentioned.

\section{Author details}


1.Research Laboratory of Spine Degenerative Disease, The Second Affiliated Hospital of Guangzhou University of Chinese Medicine, Guangzhou 510120, China;2. Laboratory of Osteology and Traumatology of Traditional Chinese Medicine, Lingnan Medical Research Center, Guangzhou University of Chinese Medicine, Guangzhou 510405, China; 3. Second College of Clinical Medicine, Guangzhou University of Chinese Medicine, Guangzhou 510405, China.

*Corresponding author: Dingkun Lin, Department of Orthopedic Surgery, The Second Affiliated Hospital of Guangzhou University of Chinese Medicine, No. 111 Dade Road, Guangzhou, Guangdong, 510120, China Email: lindingkuntcm@126.com》

\# The co-first authors: Dan Luo and Xing Li.

\section{References}

1. Jain A, Kim YT, McKeon RJ, Bellamkonda RV. In situ gelling hydrogels for conformal repair of spinal cord defects, and local delivery of BDNF after spinal cord injury. BIOMATERIALS 2006;27:497-504

2. Saunders LL, Clarke A, Tate DG, Forchheimer M, Krause JS. Lifetime prevalence of chronic health conditions among persons with spinal cord injury. Arch Phys Med Rehabil 2015;96:673-679

3. Chen WK, Feng LJ, Liu QD, et al. Inhibition of leucine-rich repeats and calponin homology domain containing 1 accelerates microglia-mediated neuroinflammation in a rat traumatic spinal cord injury model. J Neuroinflammation 2020;17:202

4. Lopez-Serrano C, Santos-Nogueira E, Francos-Quijorna I, et al. Lysophosphatidic acid receptor type 2 activation contributes to secondary damage after spinal cord injury in mice. BRAIN BEHAV IMMUN 2019;76:258-267

5. Bartanusz V, Jezova D, Alajajian B, Digicaylioglu M. The blood-spinal cord barrier: morphology and clinical implications. ANN NEUROL 2011;70:194-206

6. Reinhold AK, Rittner HL. Barrier function in the peripheral and central nervous system-a review. Pflugers Arch 2017;469:123-134

7. Yao Y, Xu J, Yu T, et al. Flufenamic acid inhibits secondary hemorrhage and BSCB disruption after spinal cord injury. THERANOSTICS 2018;8:4181-4198

8. Echeverry S, Shi XQ, Rivest S, Zhang J. Peripheral nerve injury alters blood-spinal cord barrier functional and molecular integrity through a selective inflammatory pathway. $\mathrm{J} \mathrm{NEUROSCl}$ 2011;31:10819-10828

9. Rocha LA, Sousa RA, Learmonth DA, Salgado AJ. The Role of Biomaterials as Angiogenic Modulators of Spinal Cord Injury: Mimetics of the Spinal Cord, Cell and Angiogenic Factor Delivery Agents. FRONT PHARMACOL 2018;9:164

10. Lee JY, Choi HY, Baik HH, et al. Cordycepin-enriched WIB-801C from Cordyceps militaris improves functional recovery by attenuating blood-spinal cord barrier disruption after spinal cord injury. $\mathrm{J}$ ETHNOPHARMACOL 2017;203:90-100 
11. Winkler EA, Sengillo JD, Sullivan JS, et al. Blood-spinal cord barrier breakdown and pericyte reductions in amyotrophic lateral sclerosis. ACTA NEUROPATHOL 2013;125:111-120

12. Liu $X$, Zhou $X$, Yuan W. The angiopoietin1-Akt pathway regulates barrier function of the cultured spinal cord microvascular endothelial cells through Eps8. EXP CELL RES 2014;328:118-131

13. Ge S, Pachter JS. Isolation and culture of microvascular endothelial cells from murine spinal cord. J NEUROIMMUNOL 2006;177:209-214

14. Zheng B, Zhou Y, Zhang H, et al. DI-3-n-butylphthalide prevents the disruption of blood-spinal cord barrier via inhibiting endoplasmic reticulum stress following spinal cord injury. INT J BIOL SCI 2017;13:1520-1531

15. Zheng G, Zheng F, Luo Z, et al. CO-Releasing Molecule (CORM)-3 Ameliorates Spinal Cord-Blood Barrier Disruption Following Injury to the Spinal Cord. FRONT PHARMACOL 2020;11:761

16. Jiao X, Yu Y, Meng J, et al. Dual-targeting and microenvironment-responsive micelles as a gene delivery system to improve the sensitivity of glioma to radiotherapy. ACTA PHARM SIN B 2019;9:381396

17. Shi Y, Zhang L, Pu H, et al. Rapid endothelial cytoskeletal reorganization enables early blood-brain barrier disruption and long-term ischaemic reperfusion brain injury. NAT COMMUN 2016;7:10523

18. Pan $P$, Zhao H, Zhang $X$, et al. Cyclophilin a signaling induces pericyte-associated blood-brain barrier disruption after subarachnoid hemorrhage. J Neuroinflammation 2020;17:16

19. Han JY, Li Q, Ma ZZ, Fan JY. Effects and mechanisms of compound Chinese medicine and major ingredients on microcirculatory dysfunction and organ injury induced by ischemia/reperfusion. Pharmacol Ther 2017; 177:146-173

20. Wu Q, Zheng K, Huang X, Li L, Mei W. Tanshinone-IIA-Based Analogues of Imidazole Alkaloid Act as Potent Inhibitors To Block Breast Cancer Invasion and Metastasis in Vivo. J MED CHEM 2018;61:10488-10501

21. Zhai X, Li C, Lenon GB, Xue C, Li W. Preparation and characterisation of solid dispersions of tanshinone IIA, cryptotanshinone and total tanshinones. ASIAN J PHARM SCI 2017;12:85-97

22. Xu QQ, Xu YJ, Yang C, et al. Sodium Tanshinone IIA Sulfonate Attenuates Scopolamine-Induced Cognitive Dysfunctions via Improving Cholinergic System. BIOMED RES INT 2016;2016:9852536

23. Liu J, Morton J, Miedzyblocki M, et al. Sodium tanshinone IIA sulfonate increased intestinal hemodynamics without systemic circulatory changes in healthy newborn piglets. Am J Physiol Heart Circ Physiol 2009;297:H1217-H1224

24. Zhu J, Xu Y, Ren G, et al. Tanshinone IIA Sodium sulfonate regulates antioxidant system, inflammation, and endothelial dysfunction in atherosclerosis by downregulation of CLIC1. EUR J PHARMACOL 2017;815:427-436

25. Li FQ, Zeng DK, Jia CL, et al. The effects of sodium tanshinone lla sulfonate pretreatment on high glucose-induced expression of fractalkine and apoptosis in human umbilical vein endothelial cells. INT J CLIN EXP MED 2015;8:5279-5286 
26. Wang L, Xiong X, Zhang X, et al. Sodium Tanshinone IIA Sulfonate Protects Against Cerebral Ischemia-reperfusion Injury by Inhibiting Autophagy and Inflammation. NEUROSCIENCE 2020;441:46-57

27. Ji B, Zhou F, Han L, et al. Sodium Tanshinone IIA Sulfonate Enhances Effectiveness Rt-PA Treatment in Acute Ischemic Stroke Patients Associated with Ameliorating Blood-Brain Barrier Damage. TRANSL STROKE RES 2017;8:334-340

28. Li X, Luo D, Hou Y, et al. Sodium Tanshinone IIA Silate Exerts Microcirculation Protective Effects against Spinal Cord Injury In Vitro and In Vivo. OXID MED CELL LONGEV 2020;2020:3949575

29. Zheng B, Ye L, Zhou Y, et al. Epidermal growth factor attenuates blood-spinal cord barrier disruption via PI3K/Akt/Rac1 pathway after acute spinal cord injury. J CELL MOL MED 2016;20:1062-1075

30. Wang H, Wu Y, Han W, et al. Hydrogen Sulfide Ameliorates Blood-Spinal Cord Barrier Disruption and Improves Functional Recovery by Inhibiting Endoplasmic Reticulum Stress-Dependent Autophagy. FRONT PHARMACOL 2018;9:858

31. Lee JY, Na WH, Choi HY, et al. Jmjd3 mediates blood-spinal cord barrier disruption after spinal cord injury by regulating MMP-3 and MMP-9 expressions. NEUROBIOL DIS 2016;95:66-81

32. Rong $Y$, Liu W, Wang J, et al. Neural stem cell-derived small extracellular vesicles attenuate apoptosis and neuroinflammation after traumatic spinal cord injury by activating autophagy. CELL DEATH DIS 2019;10:340

33. Gao W, Li J. Targeted siRNA delivery reduces nitric oxide mediated cell death after spinal cord injury. J Nanobiotechnology 2017;15:38

34. Sakurai M, Nagata T, Abe K, et al. Survival and death-promoting events after transient spinal cord ischemia in rabbits: induction of Akt and caspase3 in motor neurons. J Thorac Cardiovasc Surg 2003;125:370-377

35. Badner A, Vawda R, Laliberte A, et al. Early Intravenous Delivery of Human Brain Stromal Cells Modulates Systemic Inflammation and Leads to Vasoprotection in Traumatic Spinal Cord Injury. Stem Cells Transl Med 2016;5:991-1003

36. Watanabe S, Uchida K, Nakajima H, et al. Early transplantation of mesenchymal stem cells after spinal cord injury relieves pain hypersensitivity through suppression of pain-related signaling cascades and reduced inflammatory cell recruitment. STEM CELLS 2015;33:1902-1914

37. Wang C, Xu K, Wang Y, et al. Spinal cannabinoid receptor 2 activation reduces hypersensitivity associated with bone cancer pain and improves the integrity of the blood-spinal cord barrier. Reg Anesth Pain Med 2020;45:783-791

38. Gordh T, Chu H, Sharma HS. Spinal nerve lesion alters blood-spinal cord barrier function and activates astrocytes in the rat. PAIN 2006;124:211-221

39. Yu Q, Huang J, Hu J, Zhu H. Advance in spinal cord ischemia reperfusion injury: Blood-spinal cord barrier and remote ischemic preconditioning. LIFE SCI 2016;154:34-38

40. Lee JY, Kim HS, Choi HY, Oh TH, Yune TY. Fluoxetine inhibits matrix metalloprotease activation and prevents disruption of blood-spinal cord barrier after spinal cord injury. BRAIN 2012;135:2375-2389 
41. Ren J, Fu L, Nile SH, Zhang J, Kai G. Salvia miltiorrhiza in Treating Cardiovascular Diseases: A Review on Its Pharmacological and Clinical Applications. FRONT PHARMACOL 2019;10:753

42. Yang JX, Pan YY, Ge JH, et al. Tanshinone II A Attenuates TNF-alpha-Induced Expression of VCAM-1 and ICAM-1 in Endothelial Progenitor Cells by Blocking Activation of NF-kappaB. CELL PHYSIOL BIOCHEM 2016;40:195-206

43. Zhang Z, He H, Qiao Y, et al. Tanshinone IIA Pretreatment Protects H9c2 Cells against Anoxia/Reoxygenation Injury: Involvement of the Translocation of $\mathrm{Bcl}-2$ to Mitochondria Mediated by 14-3-3eta. OXID MED CELL LONGEV 2018;2018:3583921

44. Cheng J, Chen T, Li P, et al. Sodium tanshinone IIA sulfonate prevents lipopolysaccharide-induced inflammation via suppressing nuclear factor-kappaB signaling pathway in human umbilical vein endothelial cells. Can J Physiol Pharmacol 2018;96:26-31

45. Asahi M, Wang X, Mori T, et al. Effects of matrix metalloproteinase-9 gene knock-out on the proteolysis of blood-brain barrier and white matter components after cerebral ischemia. J NEUROSCI 2001;21:7724-7732

46. Remacle AG, Hullugundi SK, Dolkas J, et al. Acute- and late-phase matrix metalloproteinase (MMP)-9 activity is comparable in female and male rats after peripheral nerve injury. $\mathrm{J}$ Neuroinflammation 2018;15:89

47. Gao M, Zhang H, Trivedi A, et al. Selective Inhibition of MMP-2 Does Not Alter Neurological Recovery after Spinal Cord Injury. ACS CHEM NEUROSCI 2016;7:1482-1487

48. Kanda H, Kobayashi K, Yamanaka H, Noguchi K. COX-1-dependent prostaglandin D2 in microglia contributes to neuropathic pain via DP2 receptor in spinal neurons. GLIA 2013;61:943-956

49. Ishikawa A, Mori A, Kabei N, et al. Epidural cooling minimizes spinal cord injury after aortic crossclamping through induction of nitric oxide synthase. ANESTHESIOLOGY 2009;111:818-825

50. Pei JP, Fan LH, Nan K, et al. HSYA alleviates secondary neuronal death through attenuating oxidative stress, inflammatory response, and neural apoptosis in SD rat spinal cord compression injury. $\mathrm{J}$ Neuroinflammation 2017;14:97

51. Simard JM, Tsymbalyuk O, Ivanov A, et al. Endothelial sulfonylurea receptor 1-regulated NC Ca-ATP channels mediate progressive hemorrhagic necrosis following spinal cord injury. J CLIN INVEST 2007;117:2105-2113

\section{Figures}



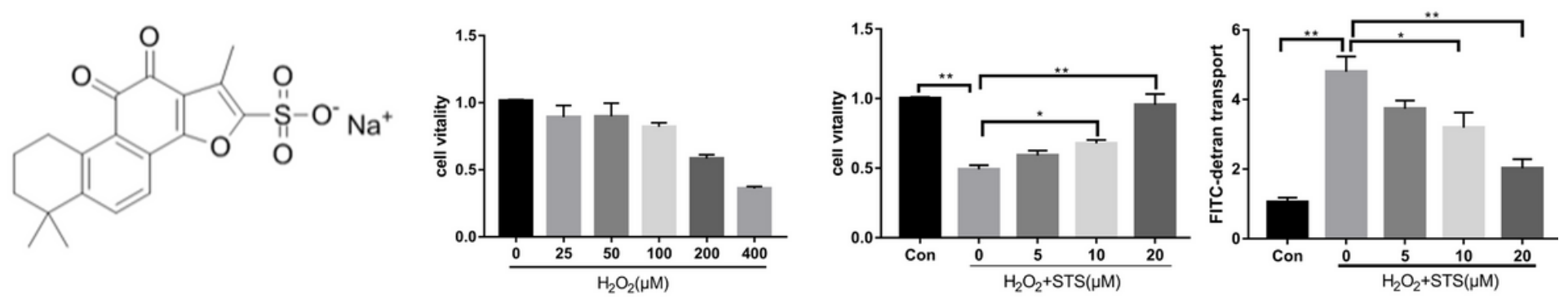

E

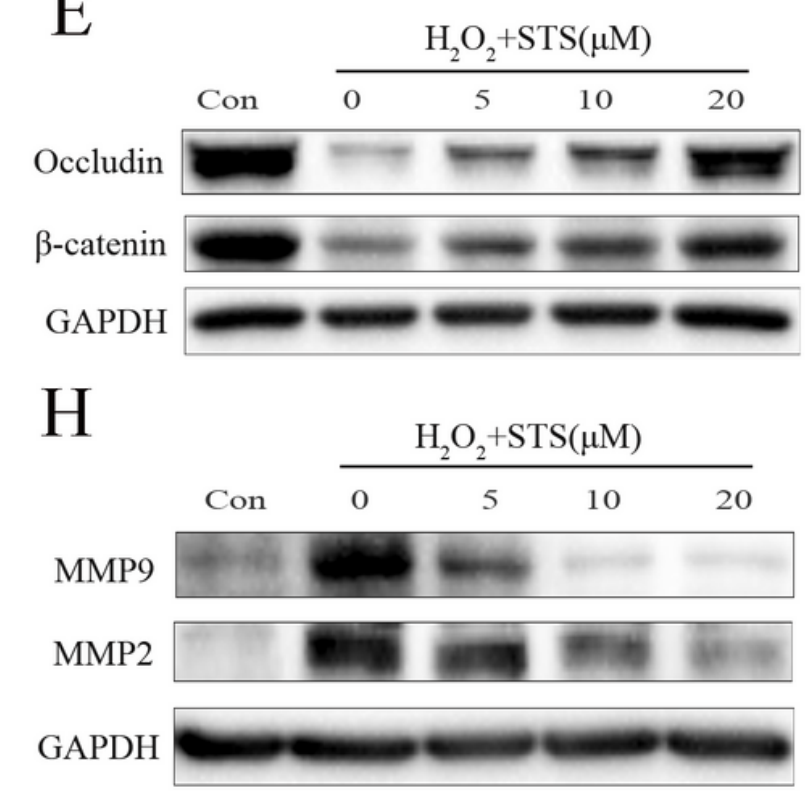

$\mathrm{F}$

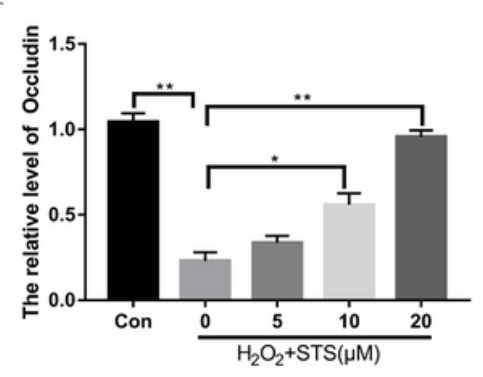

G

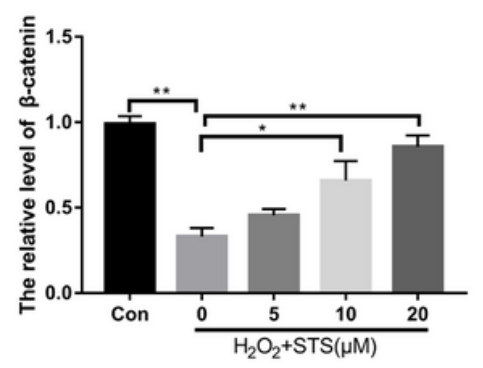

I
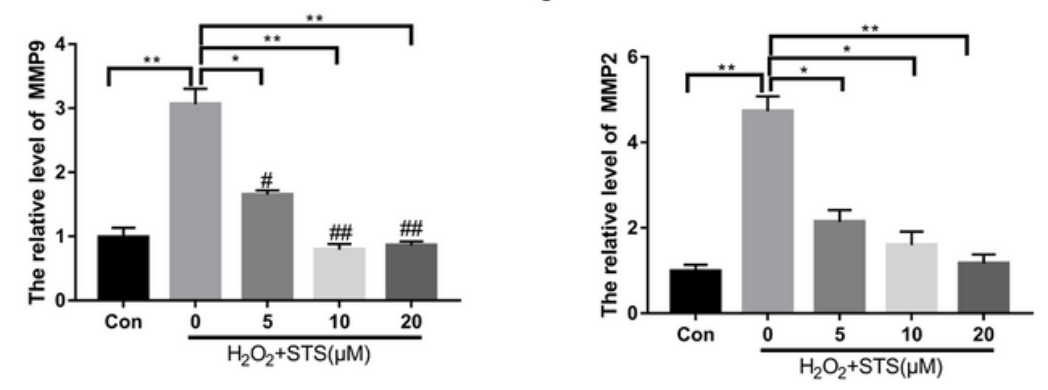

\section{Figure 1}

STS prevents loss of TJ and AJ proteins and the expression of MMP-2/MMP-9 in H2O2-treated SCMECs. (A) The molecular structure of STS. (B) Viability of SCMECs treated with different concentrations of $\mathrm{H} 2 \mathrm{O} 2$. (C) The percentage of surviving SCMECs in the indicated groups. (D) Representative fluorescence images showing FITC-dextran uptake in the differentially treated SCMECs. (E-G) Representative immunoblot showing expression levels of $\beta$-catenin and occludin in the different groups. (H-J) Representative immunoblot showing expression levels of MMP-2 and MMP-9 in SCMECs treated with or without STS under H2O2. Data indicates mean \pm SEM of three independent experiments; ${ }^{*} P<0.05$, ${ }^{\star *} P<$ 0.01 and ${ }^{* \star *} p<0.001$ by one-way ANOVA. 

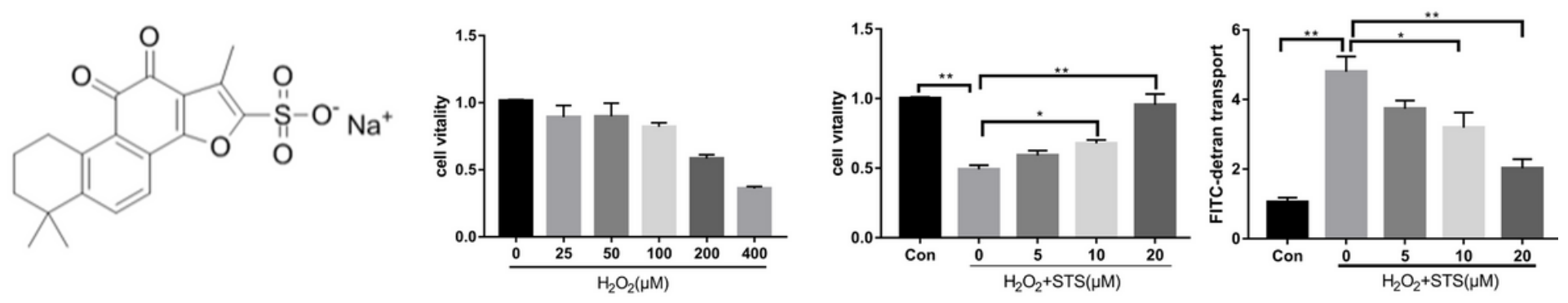

$\mathrm{E}$

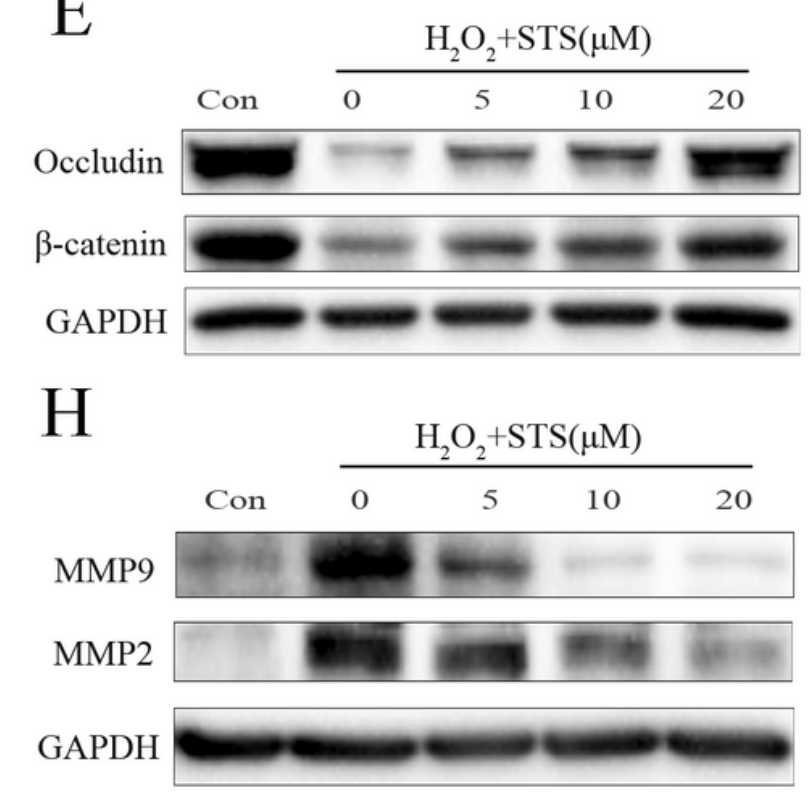

$\mathrm{F}$

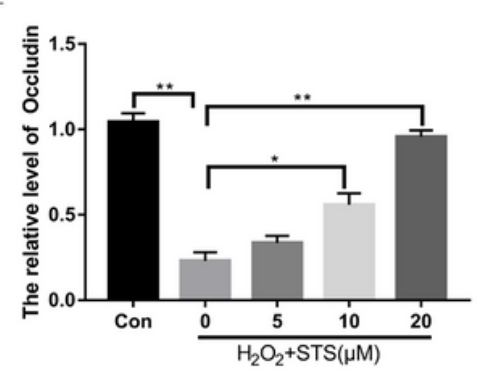

G

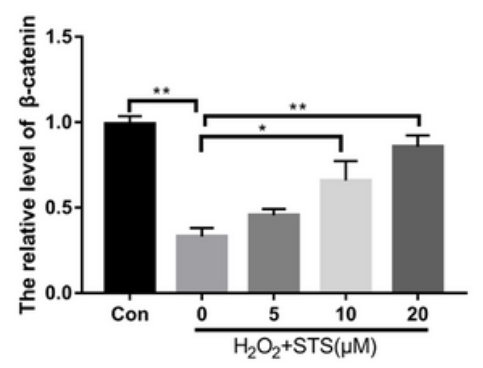

I
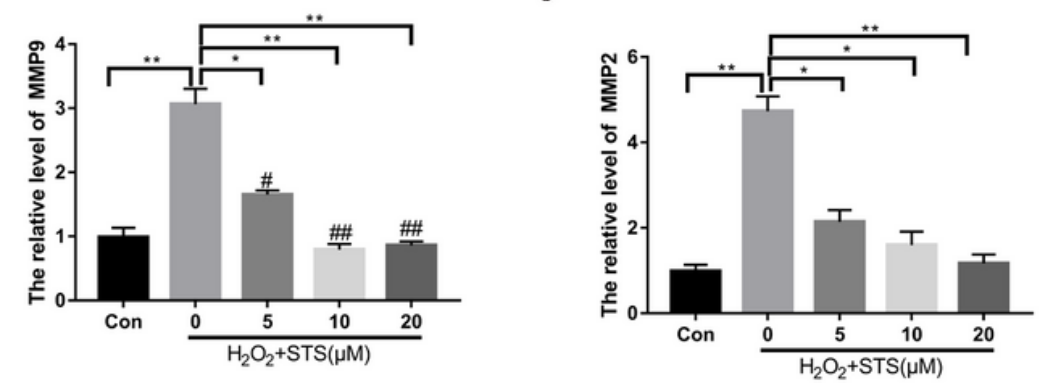

\section{Figure 1}

STS prevents loss of TJ and AJ proteins and the expression of MMP-2/MMP-9 in H2O2-treated SCMECs. (A) The molecular structure of STS. (B) Viability of SCMECs treated with different concentrations of $\mathrm{H} 2 \mathrm{O} 2$. (C) The percentage of surviving SCMECs in the indicated groups. (D) Representative fluorescence images showing FITC-dextran uptake in the differentially treated SCMECs. (E-G) Representative immunoblot showing expression levels of $\beta$-catenin and occludin in the different groups. (H-J) Representative immunoblot showing expression levels of MMP-2 and MMP-9 in SCMECs treated with or without STS under H2O2. Data indicates mean \pm SEM of three independent experiments; ${ }^{*} P<0.05$, ${ }^{\star *} P<$ 0.01 and ${ }^{* \star *} p<0.001$ by one-way ANOVA. 
A
Con

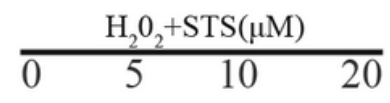

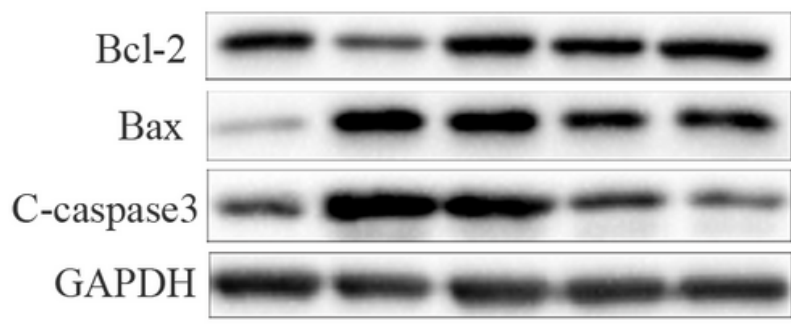

$\mathrm{B}$

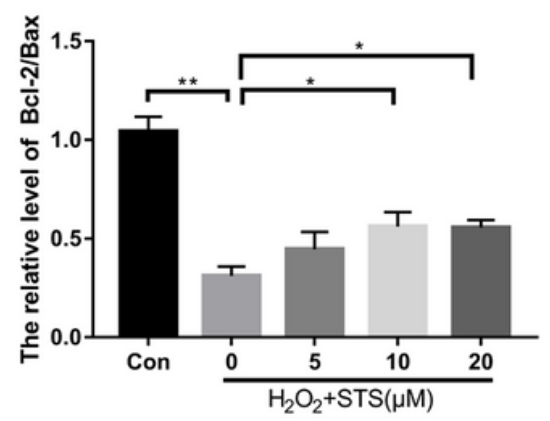

$\mathrm{C}$

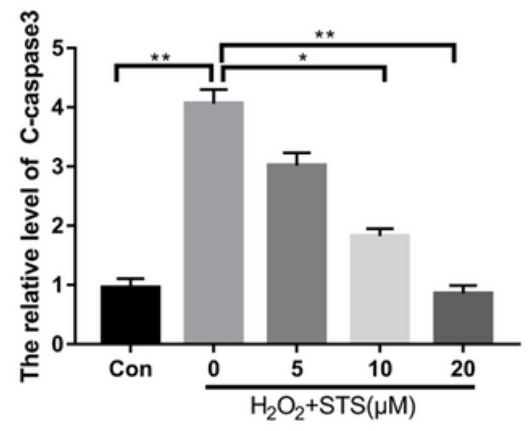

D

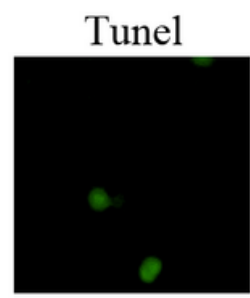

DAPI

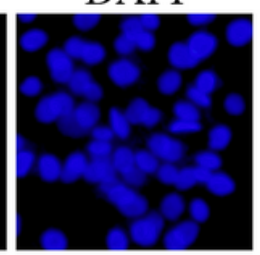

Merge

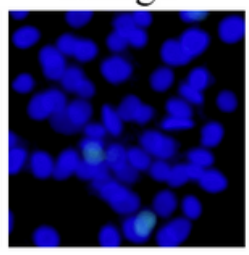

OGD
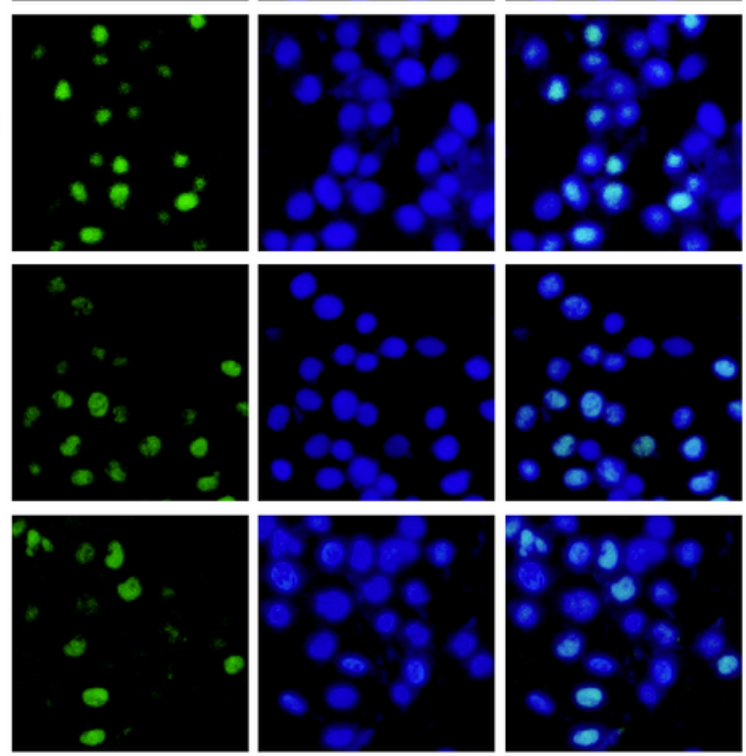

$\mathrm{H}_{2} \mathrm{O}_{2}+\mathrm{STS}(20 \mu \mathrm{M})$
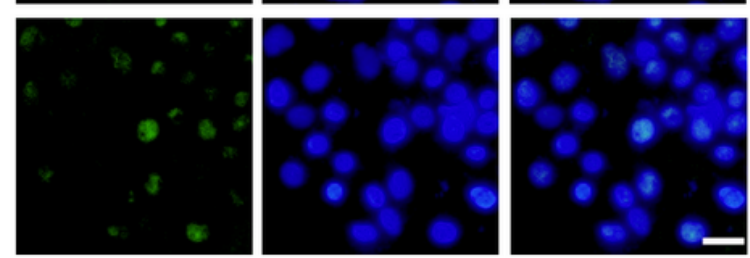

\section{Figure 2}

STS reduces apoptosis in H2O2-treated SCMECs. (A) Representative immunoblot showing expression levels of Bcl-2, Bax and C-caspase 3 in the indicated groups. (B, C) Quantification of protein bands with GAPDH as the loading control. (D) Representative immunofluorescence images of TUNEL (green)-stained SCMECs treated as indicated. Scale bar $=25 \mu \mathrm{m}$. Data indicates mean \pm SEM of three independent experiments. ${ }^{*} P<0.05,{ }^{* * P}<0.01$ and ${ }^{* \star *} p<0.001$ by one-way ANOVA. 
A
Con

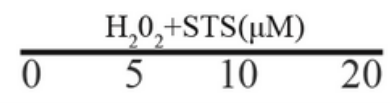

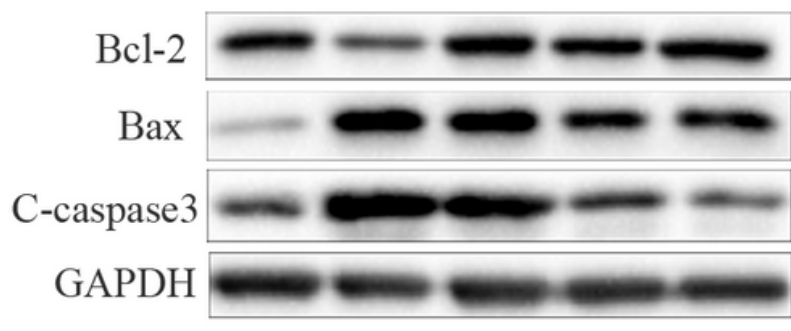

$\mathrm{B}$

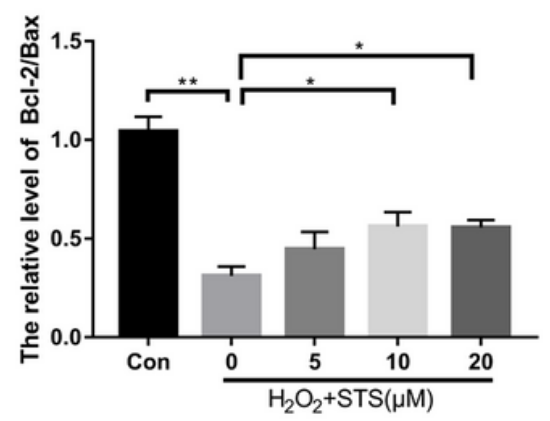

$\mathrm{C}$

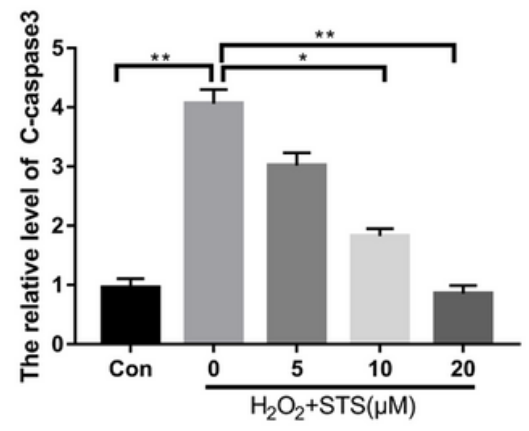

D

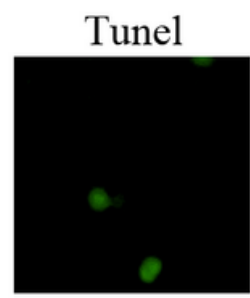

DAPI

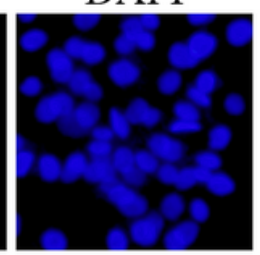

Merge

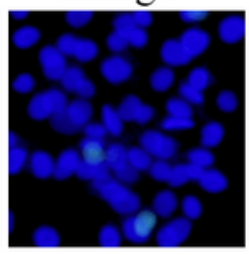

OGD
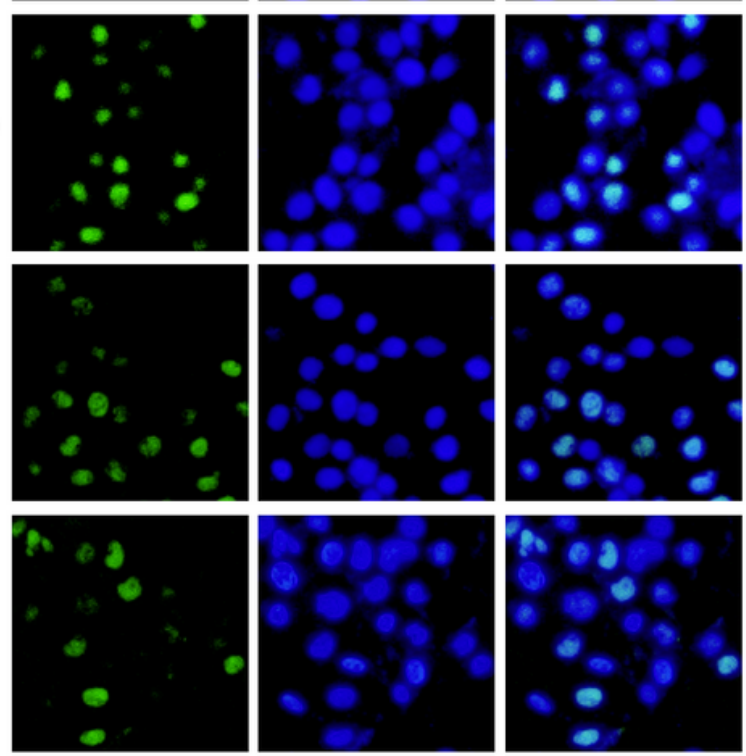

$\mathrm{H}_{2} \mathrm{O}_{2}+\mathrm{STS}(20 \mu \mathrm{M})$
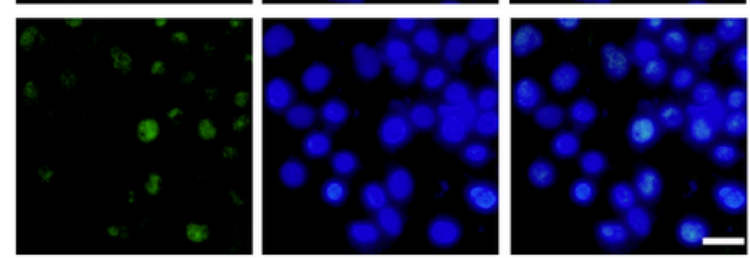

\section{Figure 2}

STS reduces apoptosis in H2O2-treated SCMECs. (A) Representative immunoblot showing expression levels of Bcl-2, Bax and C-caspase 3 in the indicated groups. (B, C) Quantification of protein bands with GAPDH as the loading control. (D) Representative immunofluorescence images of TUNEL (green)-stained SCMECs treated as indicated. Scale bar $=25 \mu \mathrm{m}$. Data indicates mean \pm SEM of three independent experiments. ${ }^{*} P<0.05,{ }^{* * P}<0.01$ and ${ }^{* \star *} p<0.001$ by one-way ANOVA. 


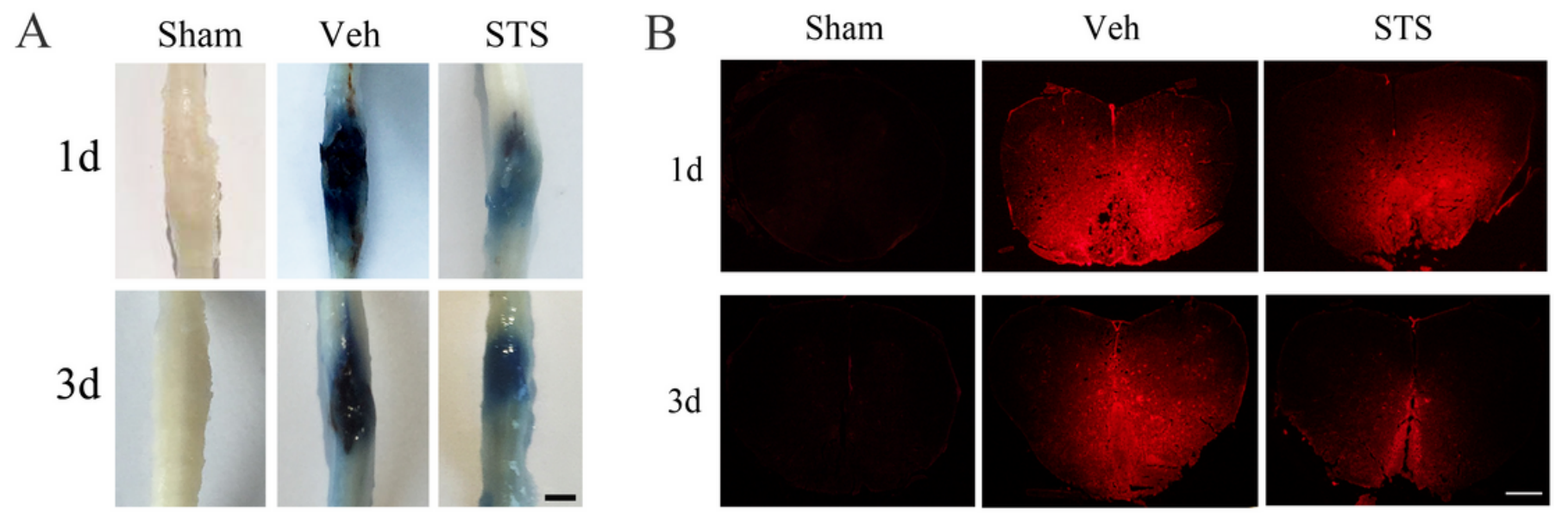

C
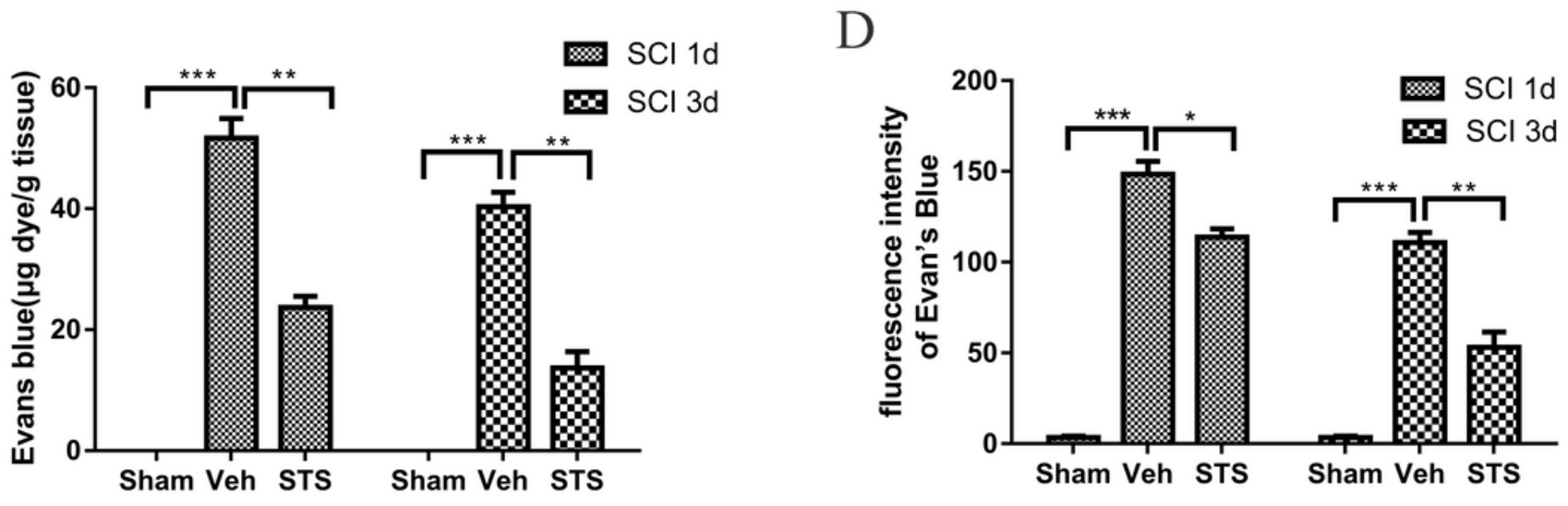

E
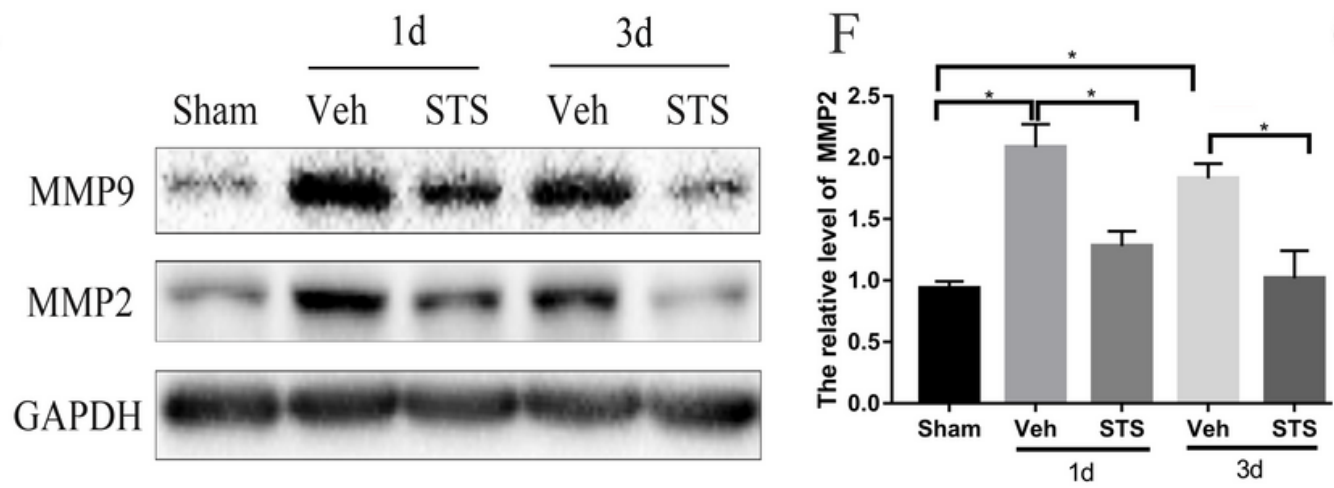

G

\section{Figure 3}

STS inhibits BSCB disruption and MMP-2/MMP-9 expression after SCI. (A) Representative images of whole spinal cords taken on days 1 and 3 post-SCI induction showing Evans Blue staining. Scale bar $=1$ $\mathrm{mm}$. (B) Representative confocal images of the transverse spinal cord sections of days 1 and 3 showing Evans Blue extravasation. Scale bar $=500 \mu \mathrm{m}$. (C) Quantification of Evans Blue content in the spinal cord $(\mu \mathrm{g} / \mathrm{g})$ of the indicated groups. (D) Quantification of the fluorescence intensity of Evans Blue in each group at 1 and 3 days after SCI. (E) Representative immunoblots showing expression levels of MMP-2 and MMP-9 at 1 and 3 days after SCI. GAPDH was used as the control. $(F, G)$ Quantification of relative MMP-2 and MMP-9 protein levels. Data indicates mean \pm SEM $(n=5)$. $P<0.05, * * P<0.01$ and $\star \star \star p<0.001$ by one-way ANOVA. 


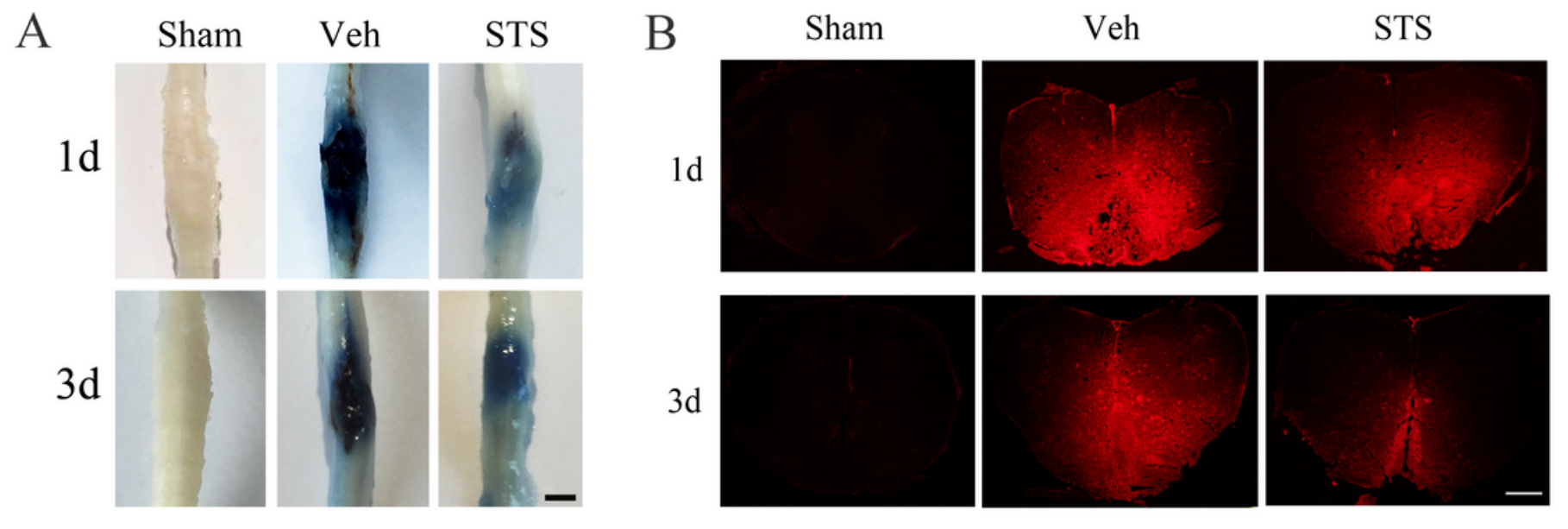

C
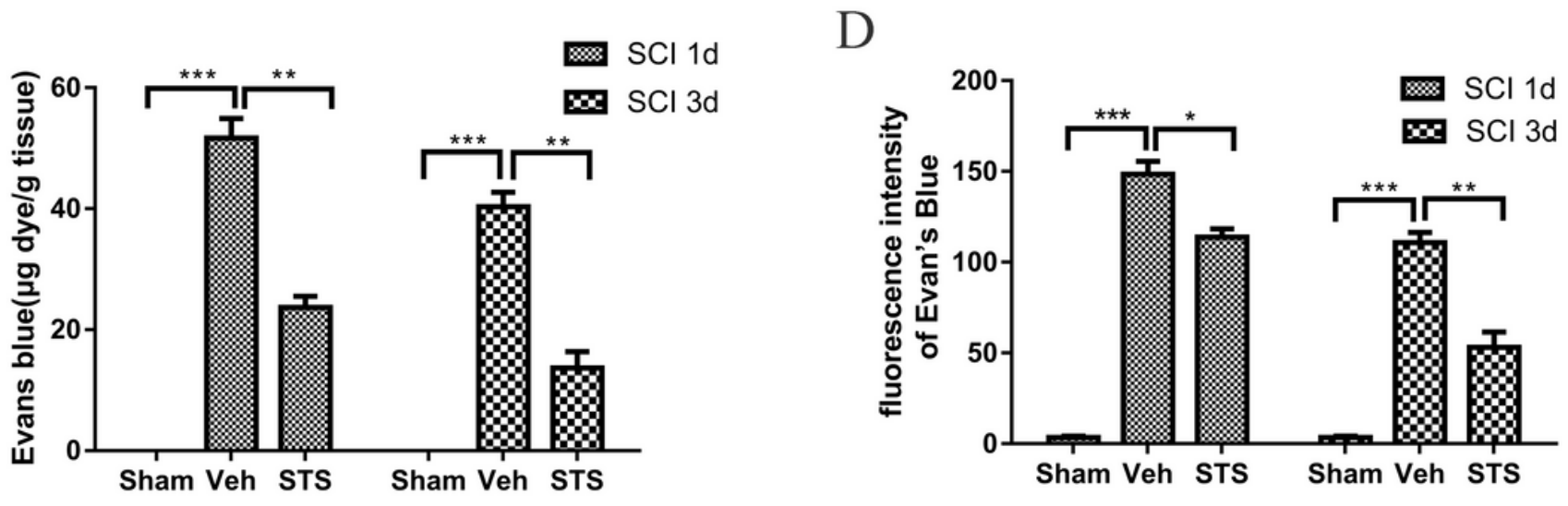

E
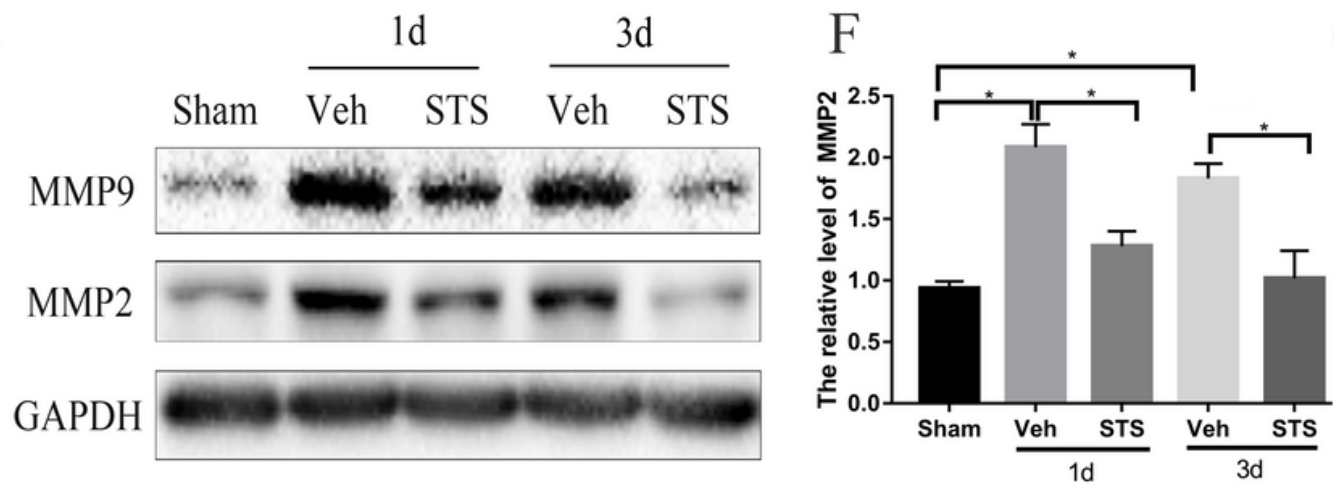

G

\section{Figure 3}

STS inhibits BSCB disruption and MMP-2/MMP-9 expression after SCI. (A) Representative images of whole spinal cords taken on days 1 and 3 post-SCI induction showing Evans Blue staining. Scale bar = 1 $\mathrm{mm}$. (B) Representative confocal images of the transverse spinal cord sections of days 1 and 3 showing Evans Blue extravasation. Scale bar $=500 \mu \mathrm{m}$. (C) Quantification of Evans Blue content in the spinal cord $(\mu \mathrm{g} / \mathrm{g})$ of the indicated groups. (D) Quantification of the fluorescence intensity of Evans Blue in each group at 1 and 3 days after SCI. (E) Representative immunoblots showing expression levels of MMP-2 and MMP-9 at 1 and 3 days after SCI. GAPDH was used as the control. $(F, G)$ Quantification of relative MMP-2 and MMP-9 protein levels. Data indicates mean \pm SEM $(n=5)$. $P<0.05, * * P<0.01$ and $\star \star \star p<0.001$ by one-way ANOVA. 


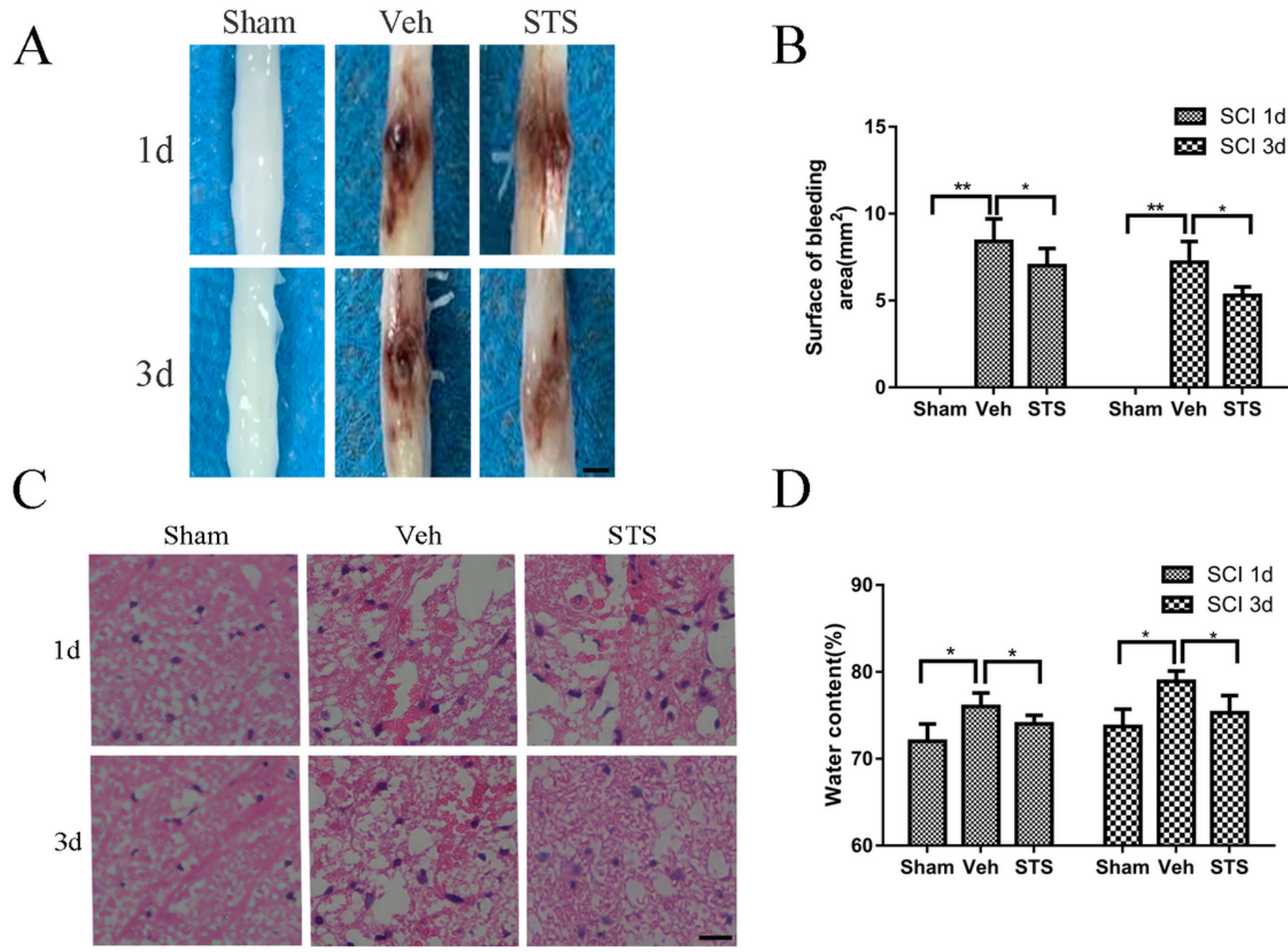

Figure 4

STS reduces secondary hemorrhage and edema. (A-B) Hemorrhagic area on the surface of injured cords from STS-treated and vehicle control mice at 1 and 3 days after injury. Scale bar $=1 \mathrm{~mm}$. (C) Representative images of HE-stained spinal cord tissues showing hemorrhage and edema. Scale bar $=50 \mu \mathrm{m}$. (D) The water content of the spinal cord in different groups at 1 and 3 days after injury. Data indicates mean values $\pm S E M, n=5$. ${ }^{*} P<0.05, * * P<0.01$ and ${ }^{\star * *} p<0.001$ by one-way ANOVA. 


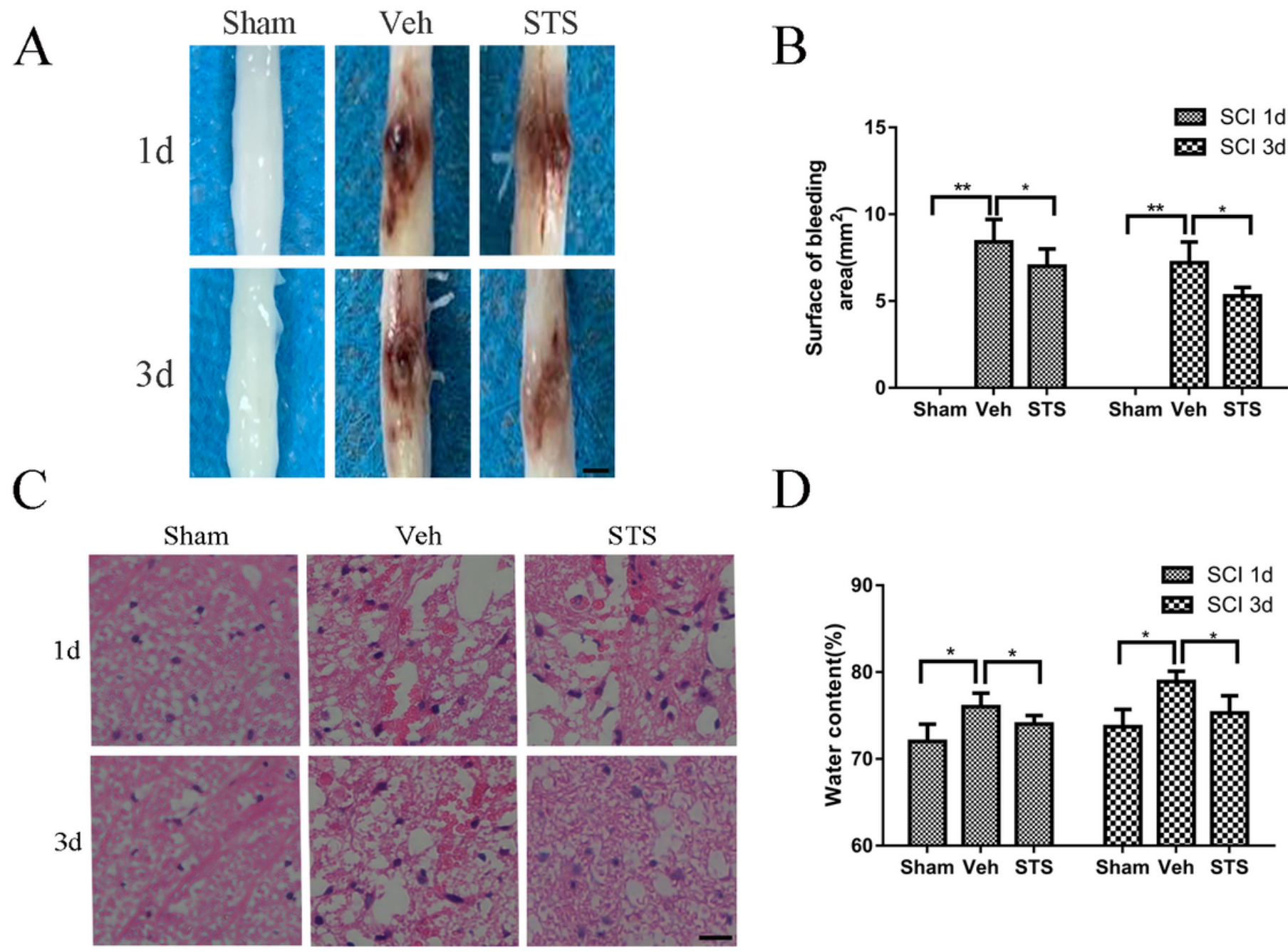

Figure 4

STS reduces secondary hemorrhage and edema. (A-B) Hemorrhagic area on the surface of injured cords from STS-treated and vehicle control mice at 1 and 3 days after injury. Scale bar $=1 \mathrm{~mm}$. (C) Representative images of HE-stained spinal cord tissues showing hemorrhage and edema. Scale bar $=50 \mu \mathrm{m}$. (D) The water content of the spinal cord in different groups at 1 and 3 days after injury. Data indicates mean values $\pm S E M, n=5$. ${ }^{*} P<0.05, * * P<0.01$ and ${ }^{\star * *} p<0.001$ by one-way ANOVA. 
A

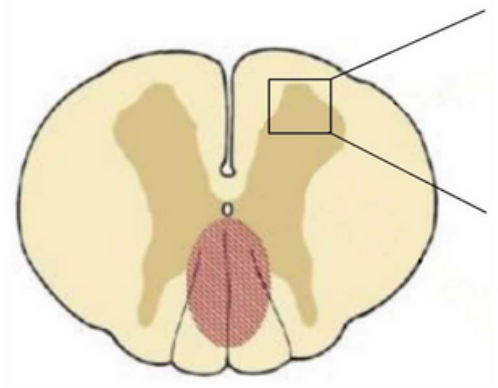

B

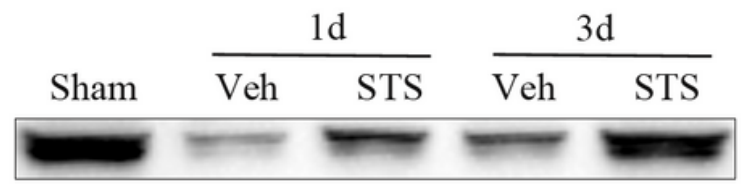

Occludin

$\beta$-catenin

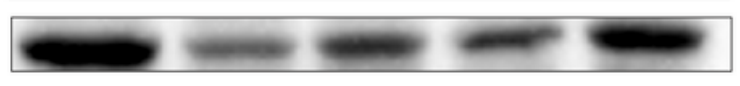

GAPDH

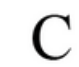

\section{$\mathrm{D}$}

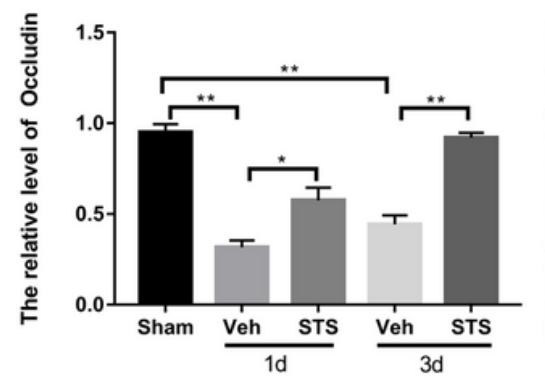

Occludin

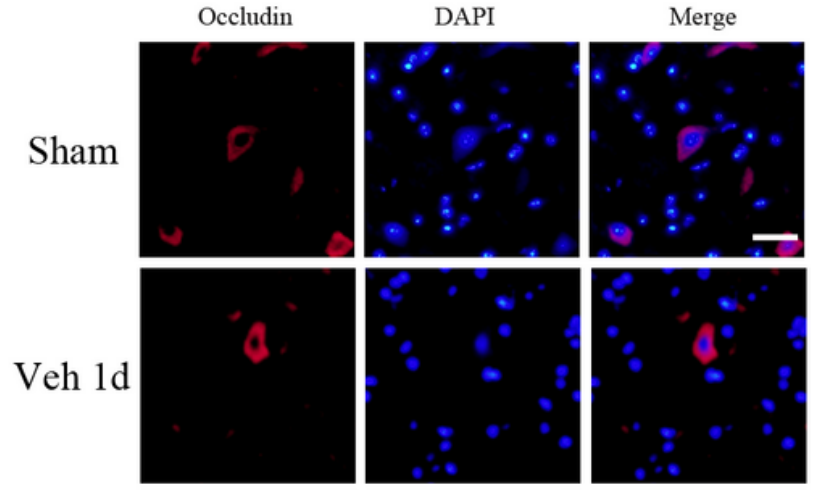

STS 1d

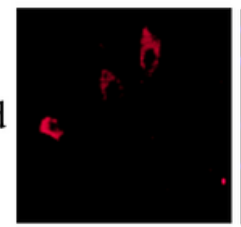

Veh 3d
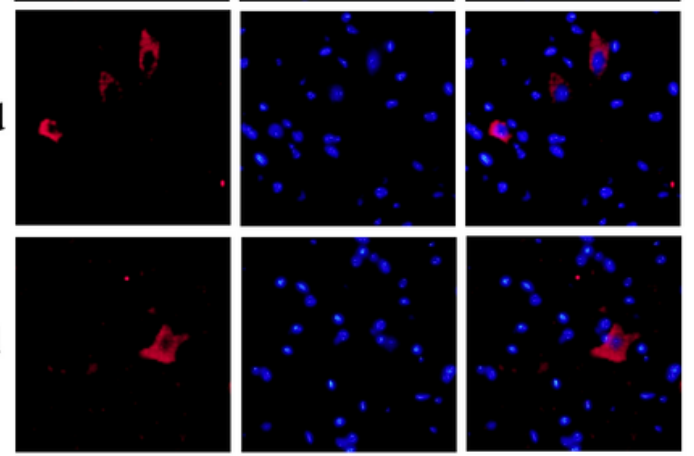

STS 3d
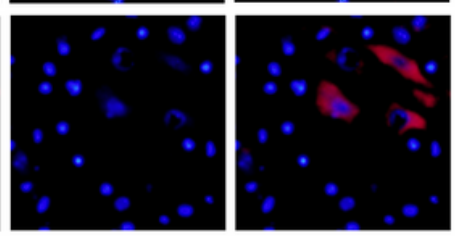

\section{Figure 5}

STS attenuates BSCB disruption by preventing loss of TJ and AJ proteins. (A) Representative images showing immunofluorescence staining of TJ protein occludin (red). Nuclei are counterstained with DAPI (blue). Scale bar $=20 \mu \mathrm{m}$. (B) Representative immunoblot showing levels of AJ protein $\beta$-catenin and occludin. (C, D) Quantification of relative protein levels. Data indicates mean $\pm S E M, n=5 .{ }^{*} P<0.05$, $* \star P$ $<0.01$ and $* * * p<0.001$ by one-way ANOVA . 
A

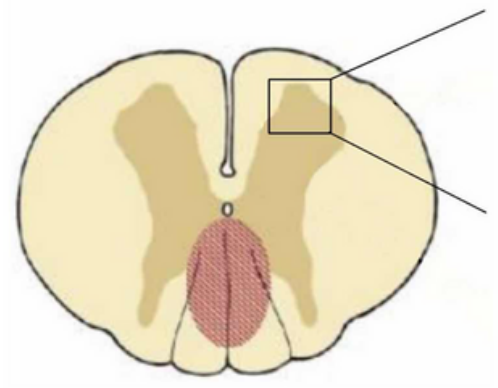

B

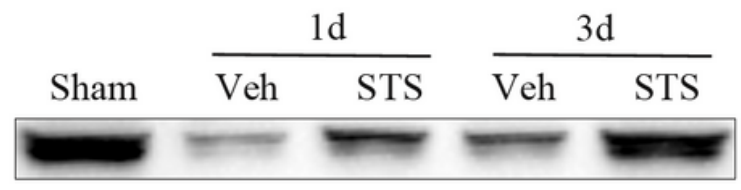

Occludin

$\beta$-catenin

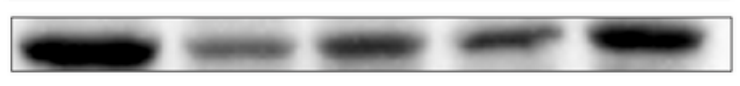

GAPDH

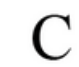

\section{$\mathrm{D}$}

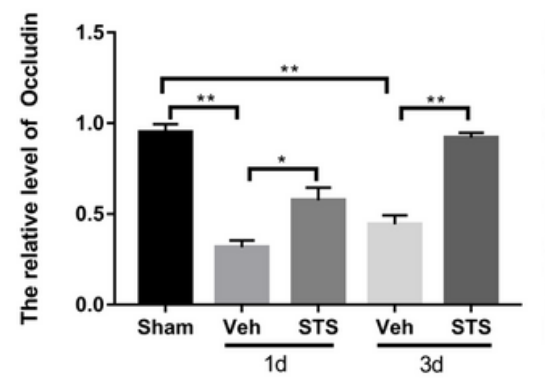

Occludin

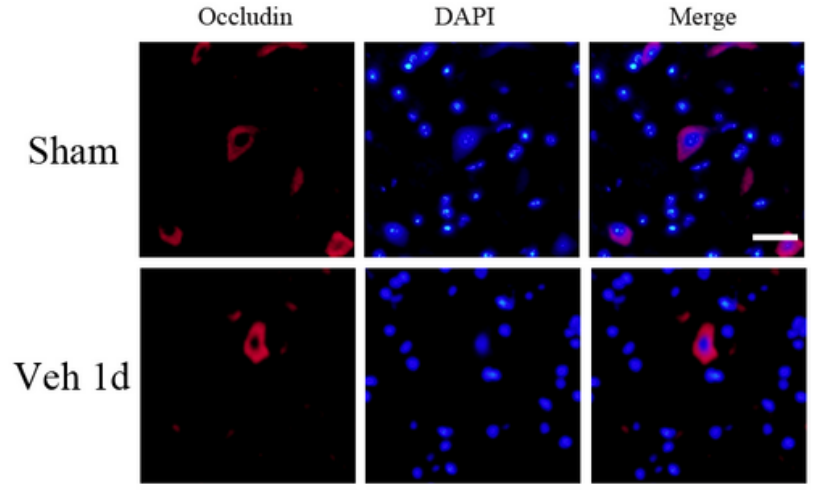

STS 1d

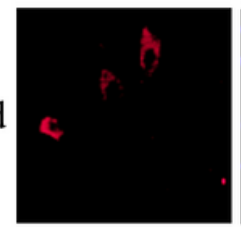

Veh 3d
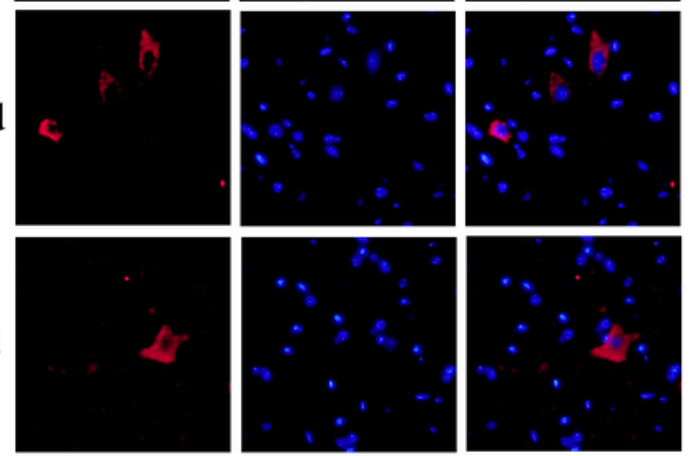

STS 3d
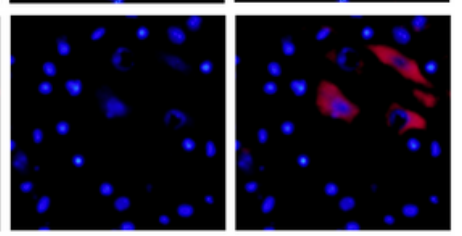

\section{Figure 5}

STS attenuates BSCB disruption by preventing loss of TJ and AJ proteins. (A) Representative images showing immunofluorescence staining of TJ protein occludin (red). Nuclei are counterstained with DAPI (blue). Scale bar $=20 \mu \mathrm{m}$. (B) Representative immunoblot showing levels of AJ protein $\beta$-catenin and occludin. (C, D) Quantification of relative protein levels. Data indicates mean $\pm S E M, n=5 .{ }^{*} P<0.05$, $* \star P$ $<0.01$ and $* * * p<0.001$ by one-way ANOVA . 


\section{Sham $\frac{1 \mathrm{~d}}{\text { Veh STS }} \frac{3 \mathrm{~d}}{\text { Veh STS }}$}

Bcl-2

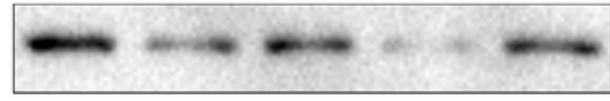

Sham

Bax

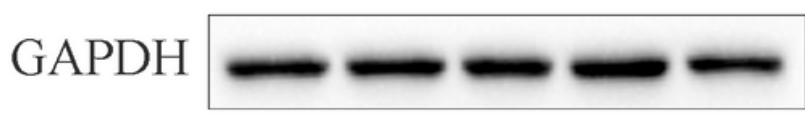

B

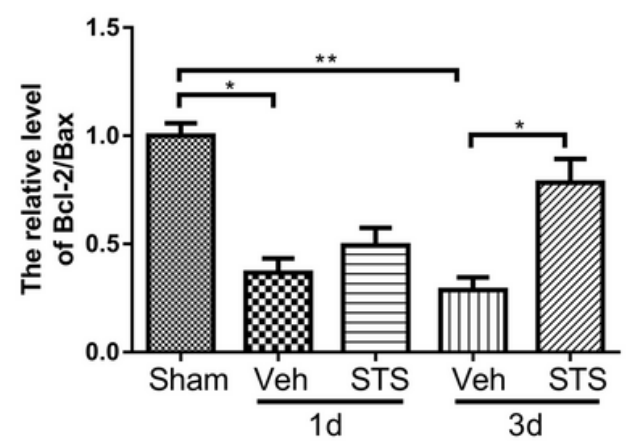

D

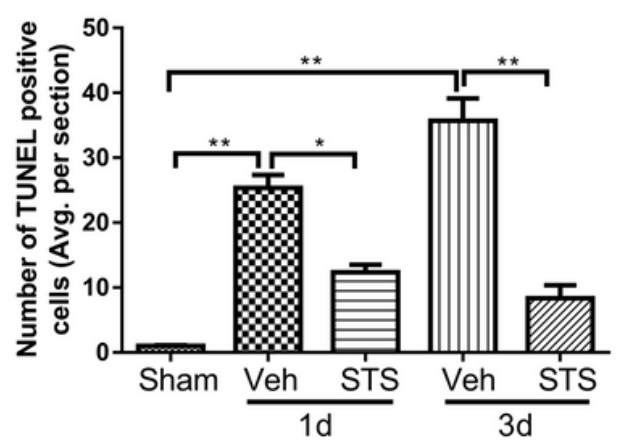

Veh 1d

STS 1d

Veh 3d
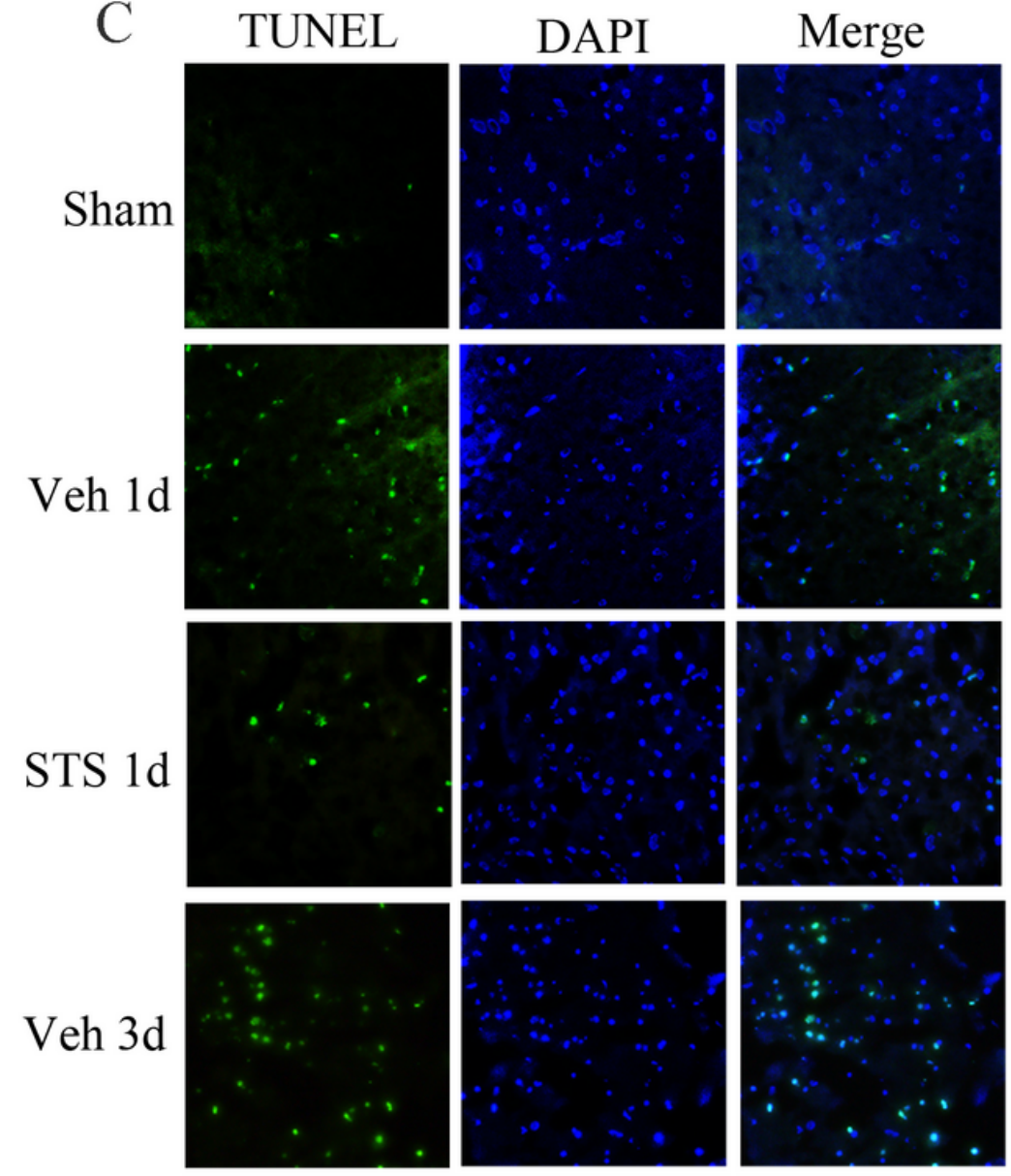

STS 3d
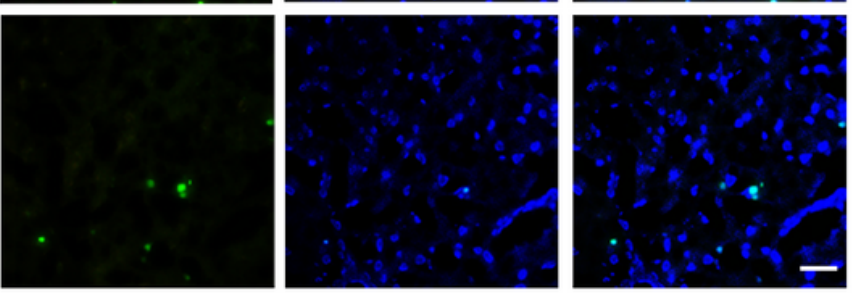

\section{Figure 6}

STS reduces apoptosis after injury. (A) Representative immunoblot showing expression levels of Bcl-2 and Bax in the spinal cord segment at the contusion epicenter at 1 and 3 days after SCI. (B)

Quantification of relative Bcl-2/Bax protein levels. (C) Representative images showing TUNEL (green)stained sections from the injured spinal cord of the indicated groups. Scale bar $=50 \mu \mathrm{m}$. (D) Quantitative estimation of apoptotic and TUNEL cells. Data indicates mean $\pm S E M, n=5 .{ }^{*} P<0.05, * * P<0.01$ and $\star \star * p<0.001$ by one-way ANOVA. 


\section{Sham $\frac{1 \mathrm{~d}}{\text { Veh STS }} \frac{3 \mathrm{~d}}{\text { Veh STS }}$}

$\mathrm{Bcl}-2$

$\operatorname{Bax}$

GAPDH

B

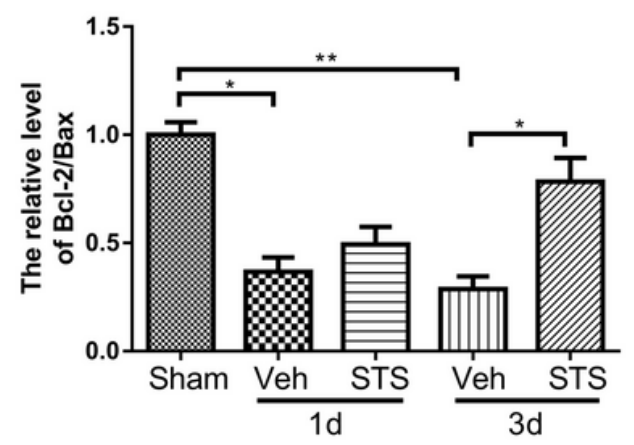

$\mathrm{D}$

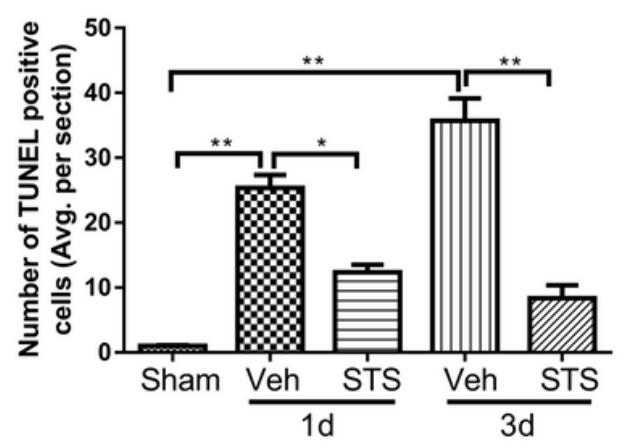

C
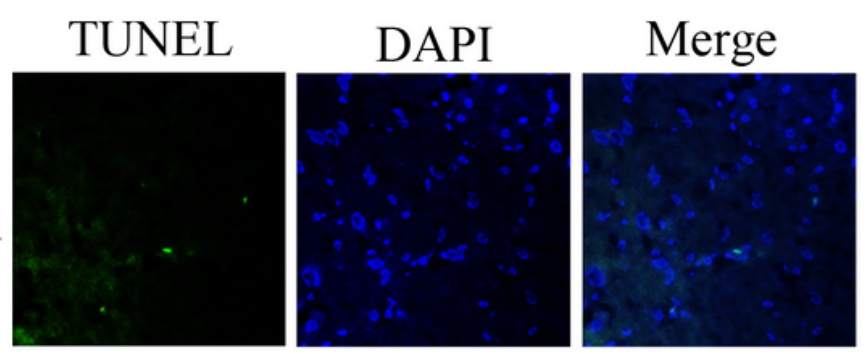

Veh 1d
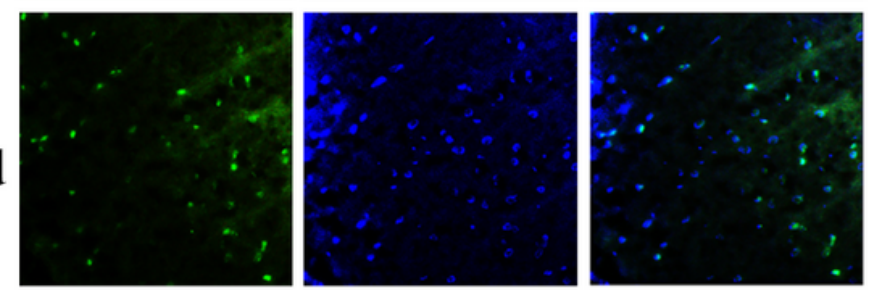

STS 1d
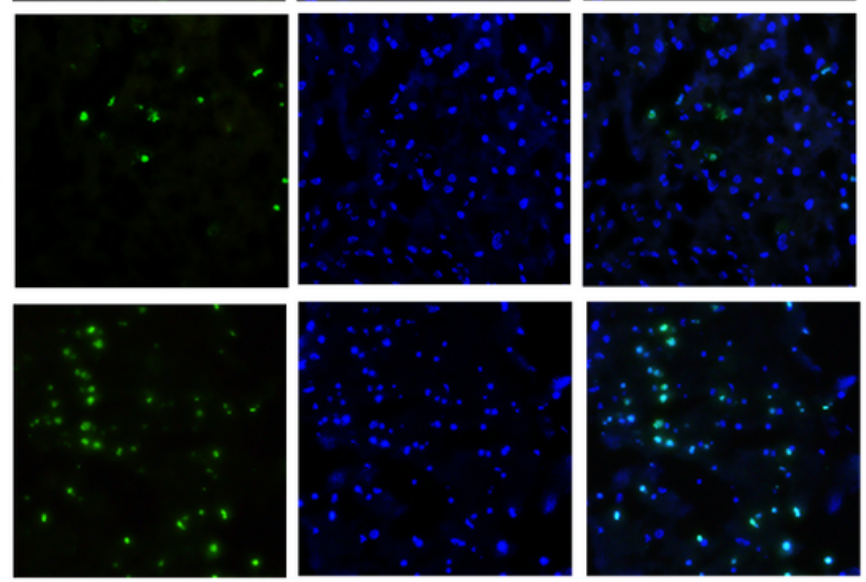

Veh 3d
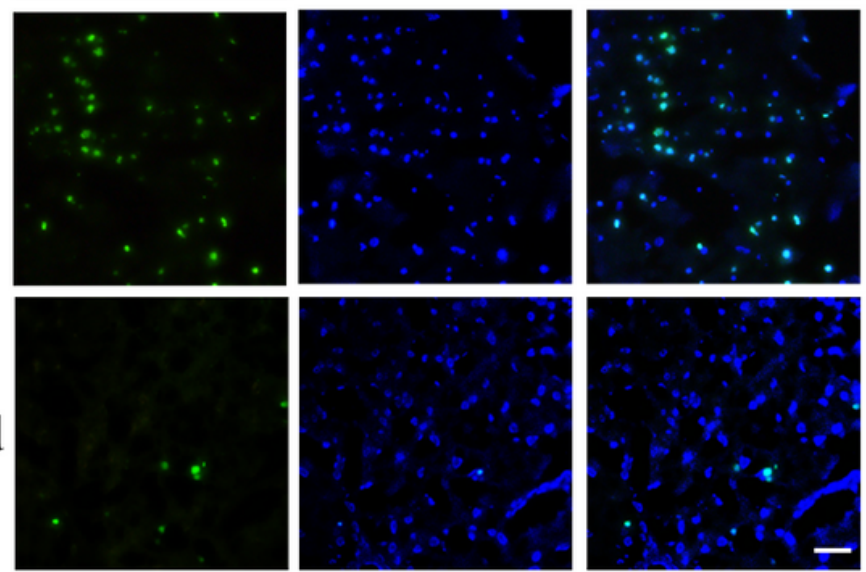

\section{Figure 6}

STS reduces apoptosis after injury. (A) Representative immunoblot showing expression levels of Bcl-2 and Bax in the spinal cord segment at the contusion epicenter at 1 and 3 days after SCI. (B)

Quantification of relative Bcl-2/Bax protein levels. (C) Representative images showing TUNEL (green)stained sections from the injured spinal cord of the indicated groups. Scale bar $=50 \mu \mathrm{m}$. (D) Quantitative estimation of apoptotic and TUNEL cells. Data indicates mean $\pm S E M, n=5 . * P<0.05,{ }^{*} P<0.01$ and $\star \star \star x<0.001$ by one-way ANOVA. 


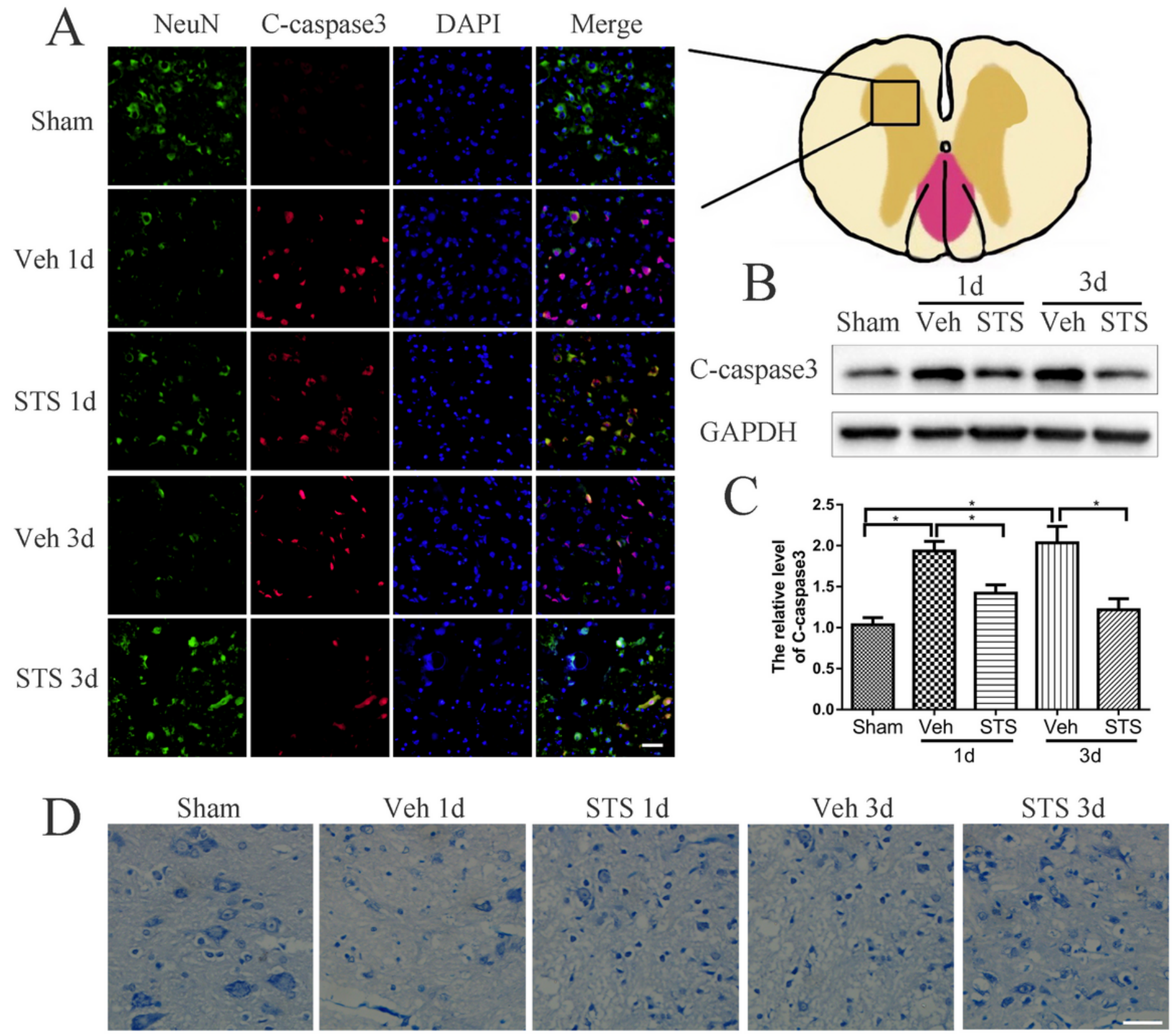

Figure 7

STS prevents neuron loss after SCl. (A) Representative immunofluorescence images showing colocalization of C-caspase3 (red) and NeuN (green) in the indicated groups. Scale bar $=50 \mu \mathrm{m}$. (B)

Representative immunoblot showing expression levels of C-caspase 3 in the spinal cord segment at the contusion epicenter. GAPDH was used as the loading control. (C) Densitometric analysis of C-caspase 3 band. (D) Representative images showing Nissl staining of spinal cord sections (scale bar $=50 \mu \mathrm{m}$ ). Data indicates mean $\pm \mathrm{SEM}, \mathrm{n}=5 .{ }^{*} \mathrm{P}<0.05,{ }^{*} \mathrm{P}<0.01$ and ${ }^{* \star *} \mathrm{p}<0.001$ by one-way ANOVA. 


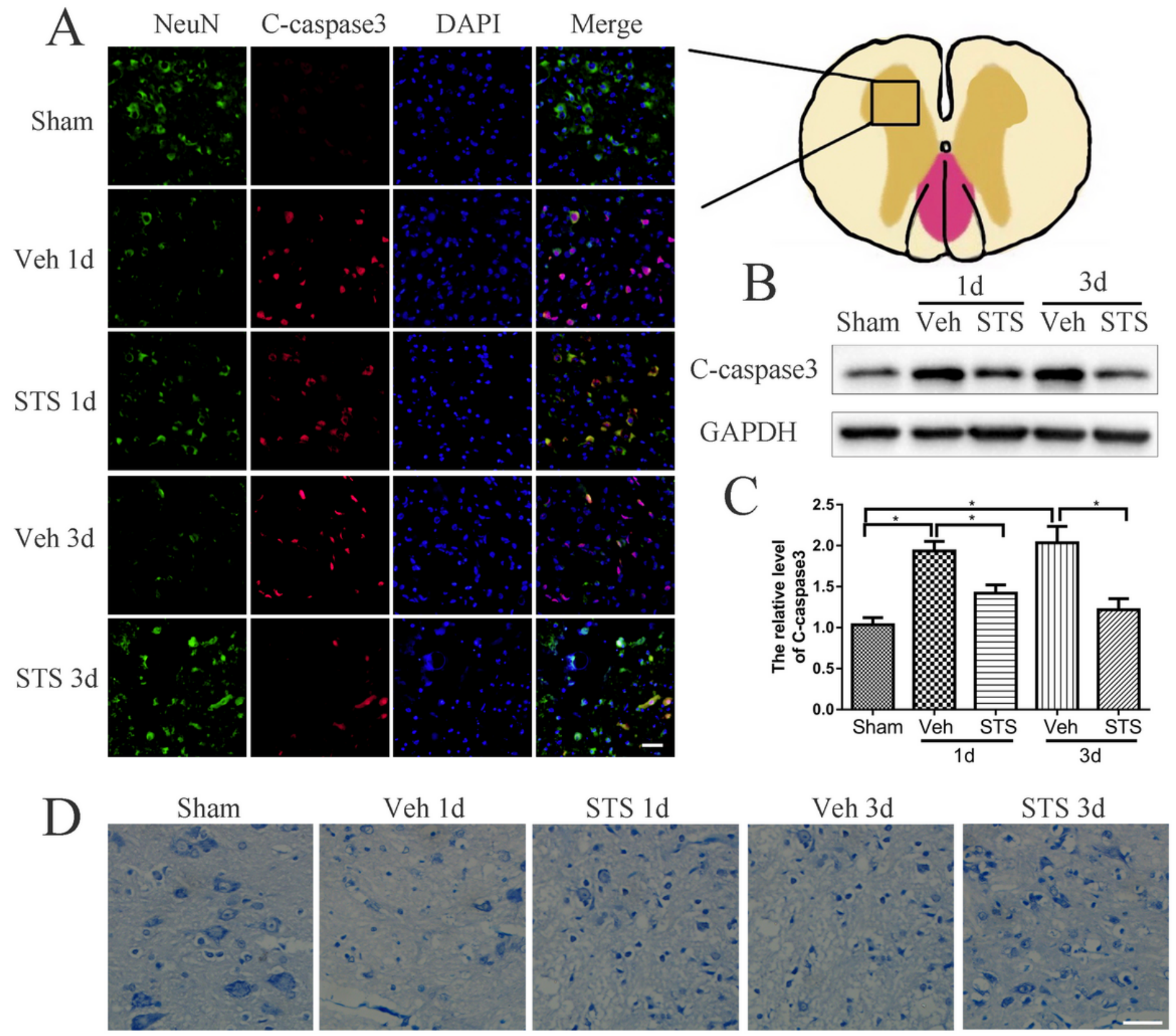

Figure 7

STS prevents neuron loss after SCl. (A) Representative immunofluorescence images showing colocalization of C-caspase3 (red) and NeuN (green) in the indicated groups. Scale bar $=50 \mu \mathrm{m}$. (B)

Representative immunoblot showing expression levels of C-caspase 3 in the spinal cord segment at the contusion epicenter. GAPDH was used as the loading control. (C) Densitometric analysis of C-caspase 3 band. (D) Representative images showing Nissl staining of spinal cord sections (scale bar $=50 \mu \mathrm{m}$ ). Data indicates mean $\pm \mathrm{SEM}, \mathrm{n}=5 .{ }^{*} \mathrm{P}<0.05,{ }^{*} \mathrm{P}<0.01$ and ${ }^{* \star *} \mathrm{p}<0.001$ by one-way ANOVA. 